\title{
On the accuracy of macroscopic equations for linearized rarefied gas flows
}

\author{
Lei $\mathrm{Wu}^{1 *}$ and Xiao-Jun Gu${ }^{2}$
}

\author{
*Correspondence: \\ wul@sustech.edu.cn \\ 'Department of Mechanics and \\ Aerospace Engineering, Southern \\ University of Science and \\ Technology, 518055 Shenzhen, \\ China \\ Full list of author information is \\ available at the end of the article
}

\begin{abstract}
Many macroscopic equations are proposed to describe the rarefied gas dynamics beyond the Navier-Stokes level, either from the mesoscopic Boltzmann equation or some physical arguments, including (i) Burnett, Woods, super-Burnett, augmented Burnett equations derived from the Chapman-Enskog expansion of the Boltzmann equation, (ii) Grad 13, regularized 13/26 moment equations, rational extended thermodynamics equations, and generalized hydrodynamic equations, where the velocity distribution function is expressed in terms of low-order moments and Hermite polynomials, and (iii) bi-velocity equations and "thermo-mechanically consistent" Burnett equations based on the argument of "volume diffusion". This paper is dedicated to assess the accuracy of these macroscopic equations. We first consider the RayleighBrillouin scattering, where light is scattered by the density fluctuation in gas. In this specific problem macroscopic equations can be linearized and solutions can always be obtained, no matter whether they are stable or not. Moreover, the accuracy assessment is not contaminated by the gas-wall boundary condition in this periodic problem. Rayleigh-Brillouin spectra of the scattered light are calculated by solving the linearized macroscopic equations and compared to those from the linearized Boltzmann equation. We find that (i) the accuracy of Chapman-Enskog expansion does not always increase with the order of expansion, (ii) for the moment method, the more moments are included, the more accurate the results are, and (iii) macroscopic equations based on "volume diffusion" do not work well even when the Knudsen number is very small. Therefore, among about a dozen tested equations, the regularized 26 moment equations are the most accurate. However, for moderate and highly rarefied gas flows, huge number of moments should be included, as the convergence to true solutions is rather slow. The same conclusion is drawn from the problem of sound propagation between the transducer and receiver. This slow convergence of moment equations is due to the incapability of Hermite polynomials in the capturing of large discontinuities and rapid variations of the velocity distribution function. This study sheds some light on how to choose/develop macroscopic equations for rarefied gas dynamics.
\end{abstract}

Keywords: Rarefied gas dynamics, Rayleigh-Brillouin scattering, Sound wave propagation, Linearized Boltzmann equation, Macroscopic equations

\section{Introduction}

A macroscopic volume of gas is a system of a very large number of molecules moving constantly in a rather irregular way. This huge number of degrees of freedom makes the trace of motion of each molecule (i.e. the microscopic description) impossible. The fundamental and practical task in the study of gas dynamics is therefore to obtain the evolution of macroscopic quantities such as the density $\rho$, flow velocity $\mathbf{u}$, temperature

(c) The author(s). 2020 Open Access This article is distributed under the terms of the Creative Commons Attribution 4.0 International License (http://creativecommons.org/licenses/by/4.0/), which permits unrestricted use, distribution, and reproduction in any medium, provided you give appropriate credit to the original author(s) and the source, provide a link to the Creative Commons license, and indicate if changes were made. 
$T$, shear stress $p_{i j}$, and heat flux q. The evolution of these macroscopic quantities may be modeled either at the mesoscopic or macroscopic level. The Boltzmann equation adopts a statistical description of dilute gas at the mesoscopic level, and its accuracy has been verified in many experiments [6]. However, due to its complicated structure, many efforts have been made to derive macroscopic equations from it to describe the rarefied gas dynamics, where the conventional Navier-Stokes (NS) equations are not valid. And it seems that the effort and desire of deriving new macroscopic equations has never stopped. In this paper we try to analyze the accuracy of major macroscopic equations proposed in the history, which may shed some light on how to choose/develop macroscopic equations. We begin with a brief introduction of the development of these equations.

Historically, according to the continuum assumption and basic conservation laws for mass, momentum and energy, macroscopic equations for $\rho$, $\mathbf{u}$ and $T$ are established in the following form:

$$
\begin{array}{r}
\frac{\partial \rho}{\partial t}+\frac{\partial\left(\rho u_{j}\right)}{\partial x_{j}}=0, \\
\frac{\partial\left(\rho u_{i}\right)}{\partial t}+\frac{\partial\left(\rho u_{i} u_{j}+p_{i j}\right)}{\partial x_{j}}=\rho a_{i}, \\
\frac{\partial(\rho E)}{\partial t}+\frac{\partial\left(\rho E u_{j}+u_{i} p_{i j}+q_{j}\right)}{\partial x_{j}}=\rho a_{j} u_{j},
\end{array}
$$

where $t$ is the time, $\mathbf{x}=\left(x_{1}, x_{2}, x_{3}\right)$ is the spatial Cartesian coordinate, $\mathbf{a}$ is the external acceleration, and $E=e+u_{i}^{2} / 2$ with $e$ being the specific internal energy that is a function of the temperature $T$. Note that the subscripts $i, j, l=1,2,3$ represent the three orthogonal spatial directions, and Einstein's summation rule is used throughout this paper.

Eq. (1) is not closed because expressions for the pressure tensor and heat flux are not known. The following phenomenological and empirical constitutive relations, i.e. Newton's law of viscosity and Fourier's law of heat conduction:

$$
\begin{array}{r}
p_{i j}=\frac{\rho k_{B} T}{m} \delta_{i j}-\mu\left(\frac{\partial u_{i}}{\partial x_{j}}+\frac{\partial u_{j}}{\partial x_{i}}\right)-\left(\zeta-\frac{2}{3} \mu\right) \frac{\partial u_{l}}{\partial x_{l}} \delta_{i j}, \\
q_{i}=-\kappa \frac{\partial T}{\partial x_{i}},
\end{array}
$$

are usually used to close Eq. (1), where $k_{B}$ is the Boltzmann constant, $m$ is the molecular mass, $\delta_{i j}$ is Kronecker delta, $\mu$ is the shear viscosity, $\zeta$ is the bulk viscosity, and $\kappa$ is the thermal conductivity. Together with the non-velocity-slip and non-temperaturejump boundary conditions, the closed NS Eqs. (1) and (2) that are established in the early 19th century have found a wide range of applications even in most of today's engineering problems.

However, the NS equations with the zero velocity-slip boundary condition cannot describe the motion of the radiometer invented by Sir William Crookes in the late 19th century. The Crookes radiometer is an airtight glass bulb, inside which there is a set of thin vanes mounted on a spindle. Each vane has a blackened side and a silvered side, which rotates when exposed to light ${ }^{1}$. The reason for this rotation was much debated following the invention of the device [39], but the currently accepted explanation for the rotation was attributed to the thermal transpiration of rarefied gas by Reynolds [53]. Specifically,

$\overline{{ }^{1} \mathrm{~A} \text { vivid illustration can be found: }}$ www.youtube.com/watch?v=r7NEI_C9Yh0. 
based on the gas kinetic theory [6, 42], Maxwell showed that an unbalanced force would be created near the edge of the heated side of the vane when inequalities of temperature exist in a gas [43].

Unlike macroscopic equations, the Boltzmann equation describes the statistical behavior of a thermodynamic system that is not in a state of equilibrium, by using the one-particle velocity distribution function (VDF) $f(t, \mathbf{x}, \mathbf{v})$, where the three-dimensional variable $\mathbf{v}=\left(v_{1}, v_{2}, v_{3}\right)$ is the molecular velocity, rather than the macroscopic flow velocity. In Boltzmann's description for dilute gases (i.e. when the mean free path of gas molecules is much larger than the molecular dimension), all molecules move in straight lines with fixed velocities until they encounter elastic collisions with other molecules. The collision is modeled by a nonlinear operator $Q\left(f, f_{*}\right)$ under the molecular chaos hypothesis, where the effect of intermolecular potential is incorporated into the collision kernel $B$. The Boltzmann equation for the time evolution of $f(t, \mathbf{x}, \mathbf{v})$ reads:

$$
\frac{\partial f}{\partial t}+\mathbf{v} \cdot \frac{\partial f}{\partial \mathbf{x}}+\mathbf{a} \cdot \frac{\partial f}{\partial \mathbf{v}}=\frac{Q\left(f, f_{*}\right)}{K n} \equiv \iint B\left(f_{*}^{\prime} f^{\prime}-f_{*} f\right) d \Omega d \mathbf{v}_{*},
$$

where the subscript $*$ represents the second molecule in the binary collision, the prime ' stands for quantities after the collision, and $\Omega$ is the solid angle. The Knudsen number $K n$ is defined as the ratio between the mean free path $\lambda$ of the gas molecules to the representative physical length scale $L$. Since for some gases ab initio intermolecular potentials have already been obtained, the Boltzmann equation contains no free parameter and is fundamental for dilute gas dynamics $[59,85]$.

When the VDF is known, macroscopic quantities can be calculated as its velocity moments, say, quantities that have clear physical meaning and are measurable are $\rho=$ $m \int f d \mathbf{v}, \mathbf{u}=(m / \rho) \int \mathbf{v} f d \mathbf{v}, p_{i j}=m \int c_{i} c_{j} f d \mathbf{v}, T=m p_{i i} / 3 k_{B} \rho$, and $q_{i}=(m / 2) \int c_{i}|\mathbf{c}|^{2} f d \mathbf{v}$, where $\mathbf{c}=\mathbf{v}-\mathbf{u}$ is the peculiar velocity. What's more, Eq. (1) can be obtained by multiplying Eq. (3) with the collision invariants $1, \mathbf{v},|\mathbf{v}|^{2}$ and integrating with respect to the molecular velocity v. However, the obtained moment system is not closed because, again, expressions for the pressure tensor and heat flux are not known. The derivation of macroscopic equations in closed forms from the Boltzmann equation is of both fundamental and practical importance, since the structure of the collision operator is complicated and hard to handle both theoretically and numerically. Yet there are huge demand of macroscopic equations that allow efficient and accurate calculation of rarefied gas dynamics with applications from aerodynamics to microfludics, and from classical to quantum/relativistic physics, e.g. in high-altitude aerothermodynamics of space vehicles [34], microelectromechanical systems [38], shale gas extractions, granular gases, radiative transfer, phonon transport, electron transport in semi-conductors, and thermal motion of Bose and Fermi gases [81]. It has also been considered as an important part in the sixth Hilbert problem: “Thus Boltzmann's work on the principles of mechanics suggests the problem of developing mathematically the limiting processes, which lead from the atomistic view to the laws of motion of continua" [32]. 


\subsection{Hilbert expansion and Chapman-Enskog expansion}

Hilbert proposed to solve the Boltzmann equation (3) via a formal asymptotic expansion of the VDF as [33]:

$$
f(t, \mathbf{x}, \mathbf{v})=\sum_{n=0}^{\infty} K n^{n} f^{(n)}(t, \mathbf{x}, \mathbf{v})
$$

where macroscopic variables such as the density, flow velocity and temperature are also expressed into the power series of $K n$; for instance, the density is $\rho=\sum_{n=0}^{\infty} K n^{n} \rho^{(n)}$ with $\rho^{(n)}=m \int f^{(n)} d \mathbf{v}$. Substituting Eq. (4) into Eq. (3) and collecting powers of $K n$ yields an infinite system of integro-differential equations for $f^{(n)}$. The zeroth-order approximation yields the Euler equations. However, NS equations never emerge according to this Hilbert expansion.

To remedy this, Chapman and Enskog proposed the famous Chapman-Enskog expansion $[10,17]$. They kept the form of Eq. (4) and the power series expansion in $K n$ for the shear stress and heat flux, i.e.

$$
\sigma_{i j}=\sum_{n=0}^{\infty} K n^{n} \sigma_{i j}^{(n)}, \quad q_{i}=\sum_{n=0}^{\infty} K n^{n} q_{i}^{(n)},
$$

but calculated $\rho, \mathbf{u}$, and $T$ only according to the zeroth-order expansion:

$$
\rho=m \int f^{(0)} d \mathbf{v}, \quad \rho \mathbf{u}=m \int \mathbf{v} f^{(0)} d \mathbf{v}, \quad T=m^{2} \int|\mathbf{c}|^{2} f^{(0)} d \mathbf{v} / 3 k_{B} \rho .
$$

With the compatibility condition $\int f^{(n)} d \mathbf{v}=\int \mathbf{v} f^{(n)} d \mathbf{v}=\int|\mathbf{c}|^{2} f^{(n)} d \mathbf{v}=0$ for $n \geq 1$, the Chapman-Enskog expansion leads to the Euler, NS, Burnett, and super-Burnett equations at the zeroth-, first, second, and third-order approximations [11], respectively.

However, the Chapman-Enskog expansion received criticisms because (i) the Burnett and super-Burnett equations are not stable to perturbation with small wavelength $[5,20]$, and (ii) one does not know "what is the status of the series of macroscopic variables obtained from the successively derived set of equations nor what step of the approximation is required or sufficient to obtain a solution that is correct up to order $K n^{n}$ " $[9,63]$, probably due to the disparity in the calculations of Eqs. (5) and (6), that is, why the density, flow velocity, and temperature are only determined by $f^{(0)}$ ? Although the augmented Burnett equations [84] solves the instability problem, the second criticism remains. Later we will show that super-Burnett equations are not necessarily more accurate than Burnett equations in the problem of dynamic light scattering, which confirms the unfavorable behavior of the successive Chapman-Enskog expansion.

\subsection{Moment methods}

Grad found an alternative way to derive macroscopic equations from the Boltzmann equation [23]. In addition to Eq. (1), he obtained macroscopic equations for the evolution of the shear stress and heat flux by respectively multiplying Eq. (3) with $c_{i} c_{j}$ and $c_{i}|\mathbf{c}|^{2}$ and integrating over $\mathbf{v}$. He then closed the moment equations by expanding the VDF into Hermite polynomials of the peculiar velocity, with the coefficients related to the considered low-order moments:

$$
f(t, \mathbf{x}, \mathbf{v})=f_{M}\left[1+\frac{\sigma_{i j}}{2 \rho R T} \frac{c_{i} c_{j}-|\mathbf{c}|^{2} \delta_{i j} / 3}{R T}+\frac{2}{5} \frac{q_{i}}{\rho(R T)^{2}} c_{i}\left(\frac{|\mathbf{c}|^{2}}{2 R T}-\frac{5}{2}\right)\right],
$$


where $R=k_{B} / m, f_{M}=(\rho / m)(2 R T)^{-3 / 2} \exp \left(-|\mathbf{c}|^{2} / 2 R T\right)$ is the Maxwellian distribution function, and $\sigma_{i j}$ is the trace-free part of the symmetrical tensor $p_{i j}$. Grad 13 (G13) equations are accurate to $K n^{2}$ [64]. Similarly, higher-order equations such as Grad 26 moments equations are constructed by adding more moments to the VDF.

The Grad moment equations are closely related to the rational extended thermodynamics equations [45], where the VDF is obtained by the maximum entropy principle, that is, the "ansatz" distribution function is an exponential function of Hermite polynomials. If 13 moments are considered, however, the exponential function becomes divergent when the peculiar velocity approaches infinity. Therefore, Taylor expansion to the first order for terms related to the heat flux is needed, and if the system deviates slightly from equilibrium, the distribution function in rational extended thermodynamics is the same as Eq. (7). When the non-equilibrium effect is strong, the polynomial in the exponent with the highest power must be even and its coefficient must be negative, see more details in Section 6.6 in the book [64] and references therein. Using the exponent ansatz for the VDF with the fourth-order Hermite polynomial, Eu developed the generalized hydrodynamic equations based on the same 13 moments used in G13 equations [1,18]. The complicated constitutive relations in Eu's equations are later simplified into the nonlinear coupled constitutive relations $[46,47]$. Since the number of moments is much less than the coefficients before Hermite polynomials, some special relations between these coefficients have to be assumed. It should also be noted that, although many numerical solutions of rarefied gas flows have been obtained by using the nonlinear coupled constitutive relations, the VDF has never been re-constructed from macroscopic quantities [35, 46, 47, 82].

With these hindsights, one may derive more new macroscopic equations in conjecture with any physical meaningful ansatz distribution functions, for example, Mott-Smith obtained normal shock wave structures by "assuming the distribution function to consist of a sum of two Maxwellian terms with temperatures and mean velocities corresponding to the subsonic and supersonic streams" [44]. In other words, the accuracy of macroscopic equations depend on how accurate the ansatz distribution functions are. This aspect will be explored in the future, but one sure thing is that macroscopic equations derived in this way may only work for certain kinds of flows, thus losing generality for describing rarefied gas flows.

Now we come back to moment equations. Although Grad moment systems are linearly stable, they are hyperbolic, which yield discontinuities in the simulation of shock waves with large Mach numbers. To remove the problem of hyperbolicity, Struchtrup and Torrihon derived the regularized 13 (R13) moment equations by combining Grad's moment method and Chapman-Enskog expansion [67]. Later, Gu and Emerson developed the regularized 26 (R26) moment equations [27]. R13 and R26 are both linearly stable, and are accurate to the order of $K n^{3}$ and $K n^{5}$, respectively.

\subsection{Macroscopic equations based on the argument of "volume diffusion"}

In all the above-mentioned macroscopic equations derived from the Boltzmann equation, the flow velocity $\mathbf{u}$ is the usual convective velocity $\mathbf{u}_{m}$. Brenner, however, proposed a bivelocity fluid model based on the argument that a deformable fluid element consisting of a large set of molecules may behave differently from a rigid body, and the motion of macroscopic fluid can occur purely diffusively by the movement of volume without a convective movement of mass $[7,8]$. Consequently, he postulated that the velocity appearing 
in Newton's viscosity law (2) should instead be the volume velocity $\mathbf{u}_{v}$. The two velocities are connected through the diffusive volume flux $\mathbf{j}_{v}$ as:

$$
\mathbf{u}_{v}=\mathbf{u}_{m}+\mathbf{j}_{v}, \quad \mathbf{j}_{v}=\alpha_{\nu} \nabla \ln \rho,
$$

where $\alpha_{v}$ is the volume diffusivity. The transport of energy is also modified correspondingly through consideration of the diffusion of internal energy. That is,

$$
q_{i}=-\kappa \frac{\partial T}{\partial x_{i}}-\alpha_{\nu} \frac{k_{B} T}{m} \frac{\partial \rho}{\partial x_{i}} .
$$

Similar idea that the fluid velocity consists of the convective and diffusion velocities has also been adopted to develop the extended NS equations $[16,55]$. The derivation of closed macroscopic equations is solely based on the argument of "volume diffusion", instead of the celebrated Boltzmann equation. Inspired by Brenner's work, Dadzie et al. proposed a Boltzmann-like gas kinetic equation and later derived the "thermo-mechanically consistent" Burnett equations [13, 15], which, unlike the Burnett equation, are linearly stable. However, this equation contains a variable which does not possess proper physical meaning. Moreover, there are some free parameters (like the volume diffusivity) which cannot be determined from low-level fundamental physical laws, but are obtained by trial-and-error [13]; this stands in sharp contrast to the Boltzmann equation where the gas dynamics can be fully determined as long as the intermolecular potential is provided.

\subsection{Method to test the accuracy of macroscopic equations}

The accuracy of many macroscopic equations have been assessed in wall-bounded problems, including the Couette, Poiseuille, thermal creep, and lid-driven cavity flows, where the rarefaction and boundary effects all play roles in the dynamics of rarefied gas. Note that the boundary conditions are not easy to construct for macroscopic equations involving higher-order derivatives/moments. Therefore, they may be not imposed in a strict manner, and whether these macroscopic equations capture the rarefaction effect or not is unclear.

Therefore, to fully assess the accuracy of macroscopic equations, we need to find a problem without the influence of gas-wall interaction. The structure of normal shock wave is one of the examples where gas-wall interaction is absent. However, due to the stability issue some macroscopic equations may not be able to produce converged solutions. Therefore, in this paper we first consider the dynamic light scattering, where light is scattered by the density fluctuation of gas molecules. It provides a perfect test bed to assess whether macroscopic equations can capture the rarefaction effect or not, since (i) this problem does not involve any gas-wall interactions, (ii) the problem is effectively onedimensional and can be linearized, which makes the macroscopic equations simple and some even allow analytical solutions, (iii) recent experimental advances have made the measurement of spectrum of dynamic light scattering very accurate, and the accuracy of linearized Boltzmann equation (LBE) has been validated; therefore the assessment will not be limited to theoretical level but have experimental evidence.

We will then assess the accuracy of macroscopic equations in the sound wave propagation problem. In fact, some macroscopic equations have already been used to calculate the speed and attenuation of sound under the periodic boundary condition, which are compared to experiments conducted between the transducer and receiver; since the gas-wall 
boundary condition (i.e. the energy accommodation coefficient) may strongly affect the pressure tensor [36], this kind of comparison cannot be used to validate the accuracy of macroscopic equations rigorously. Instead, we will compare directly with LBE solutions under the gas-kinetic boundary condition and the corresponding slip and jump boundary conditions for macroscopic equations.

The rest of the paper is organized as follows. In section 2, the spontaneous and coherent dynamic light scattering (Rayleigh-Brillouin scattering, RBS) as well as the LBE is introduced. Typical profiles of the RBS spectra in the hydrodynamic, kinetic, and freemolecular regimes are presented. In section 3, various macroscopic equations for rarefied gas flows are introduced in the linearized form and some analytical solutions for the RBS spectra are derived. The accuracy of about a dozen of macroscopic equations are analyzed by comparing the RBS spectra to those obtained from the LBE. In section 4 , the sound propagation through a rarefied gas confined between the transducer and receiver is used to test the accuracy and convergence of moment equations. Finally, conclusions are given in section 5 .

\section{Rayleigh-Brillouin scattering: solutions from the LBE}

Light propagating through the gas is scattered by the motion of gas molecules. In most general circumstances, the spectrum of the scattered light has several peaks corresponding to different scattering mechanisms. Especially, the RBS spectrum consists of the Rayleigh part due to the Doppler shift by the thermal motion of individual gas molecules and the Brillouin part related to the acoustic effect of gas molecules. Nowadays, RBS has become an invaluable non-destructive optical diagnostic technique for measuring the properties of gases, such as the sound speed, temperature, and bulk viscosity $[21,28,29,49,50,72]$ due to its large scattering cross section. One example is that the RBS is used to measure daytime atmospheric temperature [75]. Another example is that the satellite ADM-Aeolus, which has been launched in 2018 by European Space Agency to measure global wind profile from the surface of the planet to the stratosphere 30 kilometers high and hence provide much-needed information to improve weather forecasting, is based on the spontaneous RBS of rarefied gases.

\subsection{The spontaneous RBS}

In the spontaneous RBS, the incident light with a wave vector $\mathbf{k}_{i}$ is scattered due to the spontaneous fluctuation of gas density, see Fig. 1a. Suppose the angle of scattering is $\theta$, the scattering wave number is $k=\left|\mathbf{k}_{i}-\mathbf{k}_{s}\right|=2\left|\mathbf{k}_{i}\right| \sin (\theta / 2)$, and the spectrum of the scattered light is characterized by the Knudsen number, which is the ratio of the mean free path $\lambda$ of gas molecules to the scattering wavelength $L=2 \pi / k$ :

$$
K n=\frac{\lambda}{L}=\frac{\mu\left(T_{0}\right)}{p_{0} L} \sqrt{\frac{k_{B} T_{0}}{m}},
$$

where $\mu\left(T_{0}\right)$ is the shear viscosity at the gas temperature $T_{0}$, and $p_{0}$ is the average gas pressure.

Since the wavelength and frequency of light are respectively comparable to the mean free path and mean collision time of gas molecules, the spectrum of scattered light should be calculated based on the LBE [70]; a very detailed discussion on the relation of light spectrum and Boltzmann equation can be found in the Chapter 10 in Ref. [45]. For monatomic gases, suppose the scattering wave is propagating along the $x_{1}$ direction 


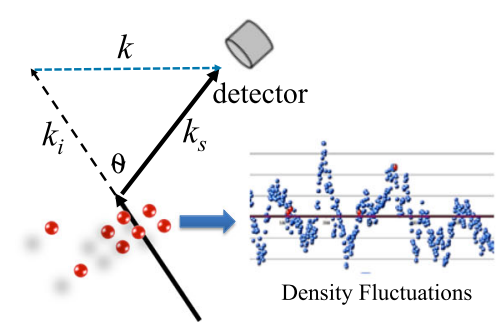

(a) Spontaneous RBS

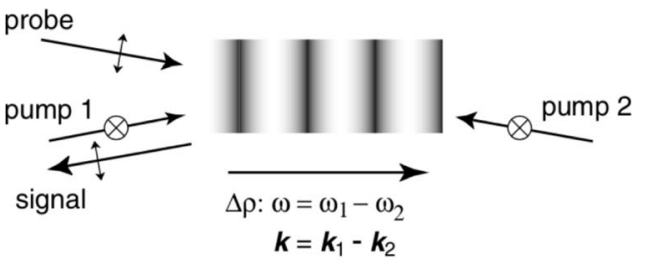

(b) Coherent RBS

Fig. 1 a Schematic of the spontaneous RBS, where the light is scattered by the spontaneous density fluctuations in the gas. b Schematic of the coherent RBS, where the light is Bragg-diffracted on the gas density fluctuations created by two pump beams. By phase matching, the signal beam follows, in reverse, the path of pump beam 1. Reprinted figure with permission from [50]. Copyright (2002) by the American Physical Society

and the gas is in the equilibrium state with a zero macroscopic velocity, the distribution function of gas molecules can be written as

$$
f\left(t, x_{1}, \mathbf{v}\right)=f_{e q}(\mathbf{v})+h\left(t, x_{1}, \mathbf{v}\right)=\frac{\exp \left(-|\mathbf{v}|^{2}\right)}{\pi^{3 / 2}}+h\left(t, x_{1}, \mathbf{v}\right)
$$

where $f_{e q}(\mathbf{v})$ is the equilibrium distribution function and $h\left(t, x_{1}, \mathbf{v}\right)$ is the VDF for small perturbation, that is, $\left|h / f_{\text {eq }}\right| \ll 1$. The evolution of the perturbation is governed by the following LBE:

$$
\frac{\partial h}{\partial t}+v_{1} \frac{\partial h}{\partial x_{1}}=\mathcal{L}_{g}(h)-v_{e q}(\mathbf{v}) h,
$$

where $\mathcal{L}_{g}(h)=\iint B\left[f_{e q}\left(\mathbf{v}^{\prime}\right) h\left(\mathbf{v}^{\prime}\right)+h\left(\mathbf{v}^{\prime}\right) f_{e q}\left(\mathbf{v}_{*}^{\prime}\right)-f_{e q}(\mathbf{v}) h\left(\mathbf{v}_{*}\right)\right] d \Omega d \mathbf{v}_{*}$ is the gain part of the linearized Boltzmann collision operator, and the equilibrium collision frequency is $v_{e q}(\mathbf{v})=\iint B f_{e q}\left(\mathbf{v}_{*}\right) d \Omega d \mathbf{v}_{*}$ [78]. The molecular velocity $\mathbf{v}$, spatial coordinate $x_{1}$, and time $t$ have been normalized by the most probable speed $v_{m}=\sqrt{2 k_{B} T_{0} / m}$, the scattering wavelength $L$, and $L / v_{m}$, respectively. Here we only consider Maxwellian gases, based on which most of the macroscopic equations are derived.

The initial perturbation in the spontaneous RBS is a density impulse $h\left(t=0, x_{1}, \mathbf{v}\right) \propto$ $\delta\left(x_{1}\right) f_{e q}(\mathbf{v})$. Applying the Laplace-Fourier transform to Eq. (12) in the temporal-spatial directions, we obtain $2 \pi i\left(f_{s}-v_{1}\right) \hat{h}=f_{e q}+\mathcal{L}_{g}(\hat{h})-v_{e q} \hat{h}$, where $f_{s}$ is the frequency shift in the scattering process normalized by the characteristic frequency $v_{m} / L$, and a hat denotes the Laplace-Fourier transform of the corresponding quantity. The spectrum of the spontaneous RBS spectrum can be determined by [70]:

$$
S_{s}\left(K n, f_{s}\right)=\Re\left(\int \hat{h} d \mathbf{v}\right),
$$

where $\Re$ is a real part of a variable, and $\hat{h}$ can be solved in the following iterative manner:

$$
\hat{h}^{j+1}(\mathbf{v})=\frac{f_{e q}(\mathbf{v})+\mathcal{L}_{g}\left(\hat{h}^{j}\right)}{2 \pi i\left(f_{s}-v_{1}\right)+v_{e q}(\mathbf{v})},
$$

where $j$ is the iteration step.

Note that the linearized Boltzmann collision operator can be solved by the fast spectral method [80]. Given the Knudsen number $K n$ and frequency shift $f_{s}$, the iteration in Eq. (14) is terminated when the relative difference in the spectrum $S_{s}$ between two consecutive iteration steps is less than $10^{-6}$. Our method can find the RBS spectrum accurately and efficiently, which has been validated by comparing with the direct simulation Monte 
Carlo method and experimental data, while the widely used Tenti S6 model [71] only works for Maxwellian molecules when the Knudsen number is small (so that the same NS equations, which can be derived both from the Boltzmann equation and S6 kinetic model by the Chapman-Enskog expansion, are valid) or very large (so that the collision operators can be neglected, and hence the Boltzmann equation and S6 kinetic model are identical), see Figure 2 in Ref. [79] and Figures 7 and 9 in Ref. [80].

It should be noted in addition to the Knudsen number defined in Eq. (10) based on the wavelength, we have another Knudsen number based on the oscillation frequency, which is defined as the ratio of the scattering frequency to the mean collision frequency $\left(\bar{v}=v_{m} / \lambda\right)$ of gas molecules:

$$
K n_{t}=\frac{f_{s} v_{m} / L}{\bar{v}}=f_{s} K n \frac{v_{m}}{\lambda \bar{v}}=f_{s} K n .
$$

Both $K n$ and $K n_{t}$ characterize the degree of non-equilibrium.

Typical spectra of the spontaneous RBS are shown in Fig. 2a. When $K n=0.001$, the gas flow is in the hydrodynamic regime, so that NS equations are valid. The spectrum consists of the central Rayleigh part near $f_{s}=0$ and two Brillouin side peaks located at $f_{s}= \pm \sqrt{\gamma R T_{0}} / v_{m}= \pm \sqrt{\gamma / 2}$, where $\gamma$ is the heat capacity ratio. Since $\sqrt{\gamma R T_{0}}$ is the sound speed, this spectrum gives a strong evidence that its Brillouin part is related to the acoustic effect of gas molecules. When $K n$ is increased to 0.01 , the position of the Brillouin peak remains unchanged, but the widths of both Rayleigh and Brillouin parts broaden, due to the increased dissipation in sound propagation. When $K n$ further increases, contributions of the central Rayleigh peak and the Brillouin side peaks to the shape of spectrum become mixed, and eventually the Brillouin parts disappear and the whole spectrum is nearly Gaussian, see the line shape when $K n=0.5$. This Gaussian line shape is predicted theoretically and observed experimentally [26, 45], in the free-molecular regime where the binary collision is negligible. We observe it here when $K n=0.5$ the gas dynamics is in strong non-equilibrium (i.e. highly rarefied) due to large values of both $K n$ and $K n_{t}$.

Finally, it should be noted that, if the gas has a macroscopic velocity $u_{g}$, the RBS spectrum will be shifted horizontally by a factor of $u_{g} / v_{m}$ in Fig. 2a. By measuring the received signals with the negative and positive frequency shifts, respectively, i.e. $\int_{-\infty}^{0} S_{s}\left(K n, f_{s}\right) d f_{s}$ and $\int_{0}^{\infty} S_{s}\left(K n, f_{s}\right) d f_{s}$, one can determine the gas velocity as long as the Knudsen number

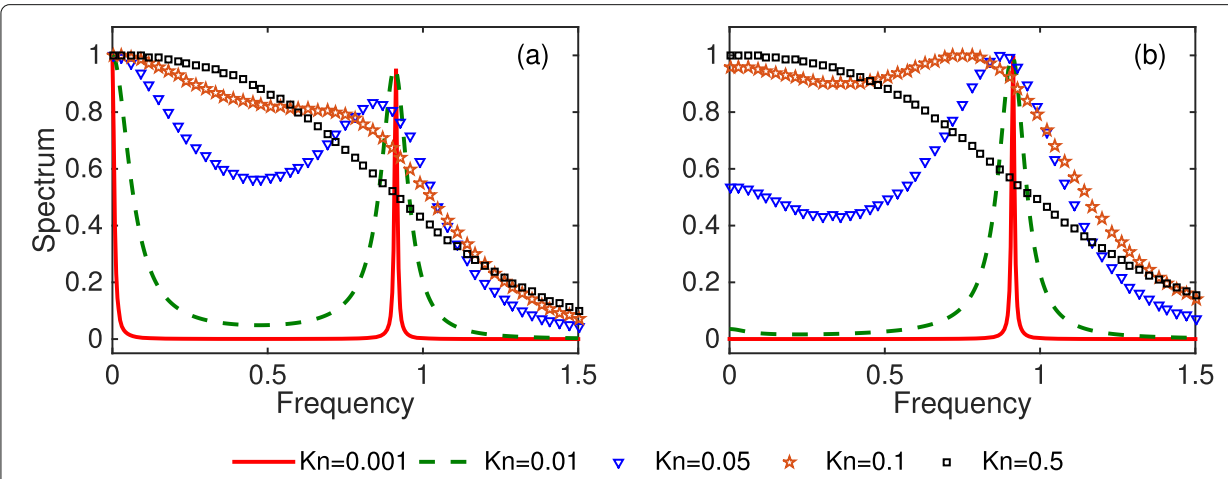

Fig. 2 Spectra of the (a) spontaneous and (b) coherent RBS from the LBE for Maxwellian gases. Note that in this figure and following figures, each spectrum has been normalized by the maximum value, where the frequency has been normalized by $v_{m} / L$. Due to the symmetry $S\left(K n, f_{s}\right)=S\left(K n,-f_{s}\right)$, only half of the spectrum is shown 
is known. This is the basic principle which will be used in the satellite ADM-Aeolus to map global wind profile. Last but not least, it has been reported that the spectrum of the dynamic light scattering for gases out of equilibrium is different to that in equilibrium [83], this may provide additional possibility to probe the VDF of gas molecules.

\subsection{The coherent RBS}

In the coherent RBS, the wavelike density perturbation is created by the moving optical lattice induced by the interference of two pump laser beams [49], see Fig. 1b. Compared to the spontaneous RBS where light is scattered everywhere, the coherent RBS has a much high signal-to-noise ratio because the scattered light only propagates in a certain direction by the phase matching method. Experimentally, efficient coherent RBS measurement on timescales of hundreds of nanoseconds has been reported by using rapidly chirped, pulsed, optical lattices [21], which "allows gas dynamic measurements in transient environments such as high-speed flows and combustion". Recently, this technique has also been used to detect in situ nanoparticles production by volumetric nanoparticle synthesis methods, with characteristic particle sizes ranging from the atomic scale to tens of nanometers [22, 61].

Suppose the wave vector $\mathbf{k}$ is propagating along the $x_{1}$ direction, the acceleration $a_{1}$ is proportional to $\cos \left[2 \pi\left(x_{1}-f_{s} t\right)\right]$ [50]. The pump beam intensities are so low that the spectrum of the scattered light can be obtained by solving the following LBE:

$$
\frac{\partial h}{\partial t}+v_{1} \frac{\partial h}{\partial x_{1}}-\cos \left[2 \pi\left(x_{1}-f_{s} t\right)\right] v_{1} f_{e q}(\mathbf{v})=\mathcal{L}_{g}(h)-v_{e q}(\mathbf{v}) h .
$$

We take the Fourier transform of Eq. (16) in both the temporal-spatial domain, and solve the resultant equation in an iterative manner. Given the frequency difference $f_{s}$ and Knudsen number $K n$, we can solve the distribution function in the following iterative manner [80]:

$$
\hat{h}^{j+1}(\mathbf{v})=\frac{v_{1} f_{e q}(\mathbf{v})+\mathcal{L}_{g}\left(\hat{h}^{j}\right)}{2 \pi i\left(f_{s}-v_{1}\right)+v_{e q}(\mathbf{v})},
$$

where $\hat{h}$ is the spatial-temporal Fourier transform of $h$.

Since in coherent RBS the spectrum is proportional to the square of density variations, the corresponding RBS spectrum is

$$
S_{c}\left(K n, f_{s}\right)=\left|\int \hat{h} d \mathbf{v}\right|^{2}
$$

Figure $2 \mathrm{~b}$ depicts typical spectra of the coherent RBS. When $K n=0.001$, the spectrum consists of the Brillouin peaks only, indicating the sound waves are resonantly pumped by the optical dipole force field. As $K n$ increases, the relative intensity of the Rayleigh peak to the Brillouin side peaks increases, and eventually the whole spectrum is Gaussian, which means that the spectrum is solely due to the random thermal motion of gas molecules, see Fig. $2 \mathrm{~b}$ when $K n=0.5$. Note that normally the gas is in the free molecular flow regime where the binary collision is negligible when $K n \gtrsim 10$, here again we observe the Gaussian spectrum (a signature of free molecular flow) when $K n=0.5$ because both the large values of the spatial and temporal Knudsen numbers are contributing to the non-equilibrium dynamics. 


\section{Rayleigh-Brillouin Scattering: accuracy of macroscopic equations}

In this section, macroscopic equations introduced in section 1 are assessed in the dynamic light scattering problem. For simplicity, dimensionless variables are introduced as follows [66]:

$$
\begin{array}{r}
\tilde{x}=\frac{x_{1}}{L_{0}}, \quad \tilde{u}=\frac{u_{1}}{v_{0}}, \quad \tilde{t}=\frac{t}{L / v_{0}}, \quad \tilde{a}=\frac{a_{1}}{v_{0}^{2} / L}, \\
\tilde{\rho}=\frac{\rho}{\rho_{0}}-1, \quad \tilde{T}=\frac{T}{T_{0}}-1, \quad \tilde{\sigma}=\frac{\sigma_{11}}{p_{0}}, \quad \tilde{q}=\frac{q_{1}}{p_{0} v_{0}},
\end{array}
$$

where $v_{0}=\sqrt{R T_{0}}$.

Eq. (1) is linearized into the following forms (tildes are omitted for clarity):

$$
\begin{array}{r}
\frac{\partial \rho}{\partial t}+\frac{\partial u}{\partial x}=0 \\
\frac{\partial u}{\partial t}+\frac{\partial \rho}{\partial x}+\frac{\partial T}{\partial x}+\frac{\partial \sigma}{\partial x}=a \\
\frac{3}{2} \frac{\partial T}{\partial t}+\frac{\partial u}{\partial x}+\frac{\partial q}{\partial x}=0
\end{array}
$$

which, as stated before, are not closed since expressions for the shear stress $\sigma$ and the heat flux $q$ are not known.

\subsection{Macroscopic equations from the Chapman-Enskog expansion}

According to the Chapman-Enskog expansion, the NS equations are derived from the Boltzmann equation to the first order of $K n$. In dynamic light scattering, the shear stress and heat flux in Eq. (20) are given by

$$
\sigma^{(N S)}=-\frac{4}{3} K n \frac{\partial u}{\partial x}, \quad q^{(N S)}=-\frac{15}{4} K n \frac{\partial T}{\partial x} .
$$

Applying the Laplace transform for the temporal variable $t$ and the Fourier transform for the spatial variable $x$ in the spontaneous RBS, or the Fourier transform in both temporal and spatial directions in the coherent RBS, Eqs. (20) and (21) are turned into the following matrix form [41]:

$$
\left[\begin{array}{ccc}
-i \varpi & 2 i \pi & 0 \\
2 i \pi & -i \varpi+\frac{16}{3} \pi^{2} K n & 2 i \pi \\
0 & 2 i \pi & -\frac{3}{2} i \varpi+15 \pi^{2} K n
\end{array}\right]\left[\begin{array}{c}
\hat{\rho} \\
\hat{u} \\
\hat{T}
\end{array}\right]=\left[\begin{array}{c}
N_{1} \\
N_{2} \\
0
\end{array}\right]
$$

where $\hat{\rho}, \hat{u}$, and $\hat{T}$ are the spectra of the perturbation density, velocity, and temperature, respectively, $i$ is the imaginary unit, and $\varpi=2 \sqrt{2} \pi f_{s}$ is the normalized angular frequency. In the spontaneous RBS we have $N_{1}=1$ due to the Laplace transform of the initial density perturbation and $N_{2}=0$, while in the coherent RBS we have $N_{1}=0$ but $N_{2}=1$ due to the presence of external acceleration from the interference of two pump beams, see Fig. 2b and Eq. (16). Spectra of the spontaneous and coherent RBS are given by $S_{s}=\Re(\hat{\rho})$ and $S_{c}=|\hat{\rho}|^{2}$, respectively. 
Solving Eq. (22) for the spectrum of the density disturbance $\hat{\rho}$, we find that the spectra for the spontaneous and coherent RBS can be described by:

$$
\begin{gathered}
S_{s}^{N S}=\Re\left(\frac{160 i K n^{2} \pi^{4}+46 K n \varpi \pi^{2}-3 i \varpi^{2}+8 i \pi^{2}}{160 K n^{2} \varpi \pi^{4}-46 i K n \varpi^{2} \pi^{2}+120 i K n \pi^{4}-3 \varpi^{3}+20 \varpi \pi^{2}}\right), \\
S_{c}^{N S}=\left|\frac{6 \pi\left(10 K n \pi^{2}-i \varpi\right)}{160 K n^{2} \varpi \pi^{4}-46 i K n \varpi^{2} \pi^{2}+120 i K n \pi^{4}-3 \varpi^{3}+20 \varpi \pi^{2}}\right|^{2} .
\end{gathered}
$$

If the Chapman-Enskog expansion is applied to the second-order of $K n$, Burnett equations can be derived. For Maxwellian molecules, the linearized constitutive relations for the shear stress and heat flux become

$$
\sigma^{(B)}=\sigma^{(N S)}-K n^{2}\left(\frac{4}{3} \frac{\partial^{2} \rho}{\partial x^{2}}-\frac{2}{3} \frac{\partial^{2} T}{\partial x^{2}}\right), \quad q^{(B)}=q^{(N S)}-\frac{7}{4} K n^{2} \frac{\partial^{2} u}{\partial x^{2}} .
$$

which leads to the following spontaneous and coherent RBS spectra:

$$
\begin{array}{r}
S_{s}^{(B)}=\Re\left(\frac{i\left(9 \varpi^{2}+138 i \varpi K n \pi^{2}-584 K n^{2} \pi^{4}-24 \pi^{2}+448 K n^{4} \pi^{6}\right)}{D^{(B)}}\right), \\
S_{c}^{(B)}=\left|\frac{\pi\left(10 \pi^{2} K n-i \varpi\right)}{D^{(B)}}\right|^{2},
\end{array}
$$

with $D^{(B)}=-60 \pi^{2} \varpi-360 i \pi^{4} K n-776 \varpi K n^{2} \pi^{4}-1920 i K n^{3} \pi^{6}+9 \varpi^{3}+138 i \varpi^{2} K n \pi^{2}+$ $448 \pi^{6} \varpi K n^{4}$.

Figure 3 shows the spectra of both spontaneous and coherent RBS. It is seen that the NS equations perform well up to $K n \approx 0.02$. Burnett equations, although accurate to the second-order of $K n$, does not seem to improve the accuracy in predicting the spectra of spontaneous RBS. However, for the coherent RBS, Burnett equations perform well up to $K n \approx 0.05$.

Super-Burnett equations can be derived if the Chapman-Enskog expansion is applied to the third-order of $K n$, where the shear stress and heat flux in this particular problem are [60]:

$$
\sigma^{(S B)}=\sigma^{(B)}+\frac{2}{9} K n^{3} \frac{\partial^{3} u}{\partial x^{3}}, \quad q^{(S B)}=q^{(B)}+K n^{3}\left(\theta_{7} \frac{\partial^{3} T}{\partial x^{3}}-\frac{5}{8} \frac{\partial^{3} \rho}{\partial x^{3}}\right),
$$

with $\theta_{7}=-157 / 16$ for Maxwellian molecules.

Like Burnett equations, super-Burnett equations are unstable to disturbance with small wavelength. Zhong et al. proposed the augmented Burnett equations [84], where they kept the nonlinear expressions for the shear stress and heat flux from the Burnett equations at the second order of $K n$, while the third-order parts are chosen from the super-Burnett equations as the corresponding linearized terms in one-dimensional problem. In this dynamic light scattering problem, expressions for stress and heat flux in the augmented Burnett equations are also given by Eq. (27). The coefficient $\theta_{7}$, however, is chosen as $\theta_{7}=11 / 16$, due to the wrong calculation [73]; this erroneous parameter happens to make the augmented Burnett equations stable.

Figure 4 illustrates the RBS spectra obtained from the super-Burnett equations with various values of $\theta_{7}$. It is surprising that the original super-Burnett equations with $\theta_{7}=$ 

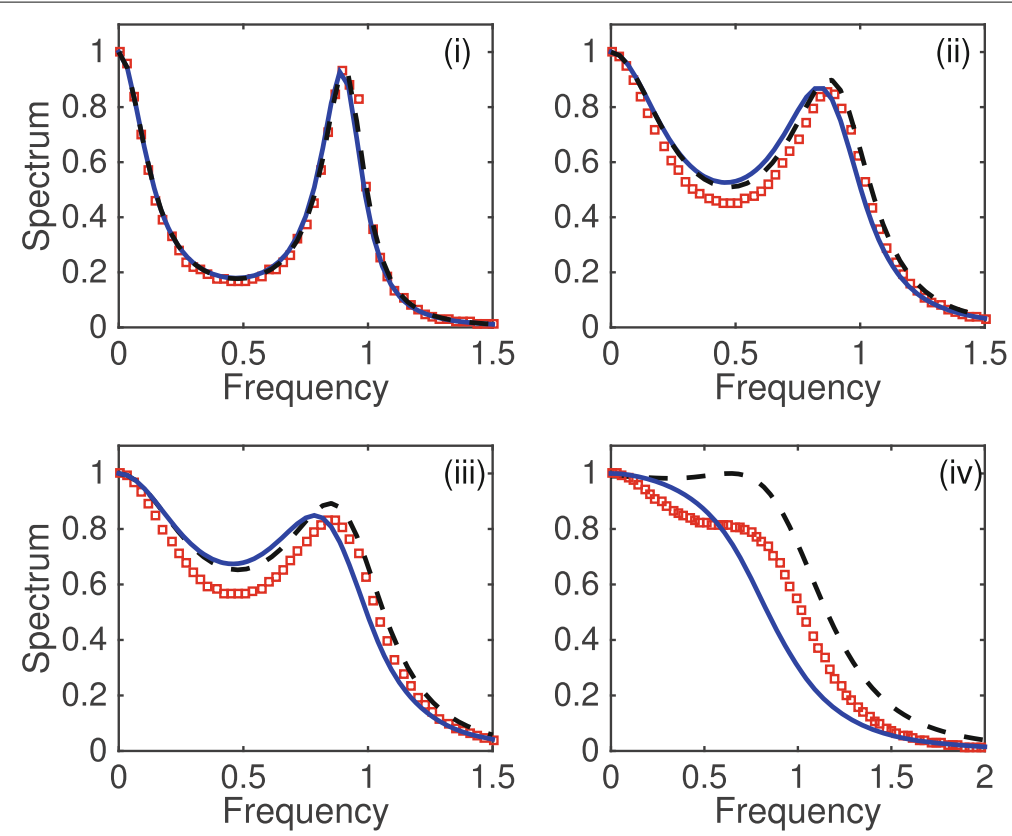

(a) Spontaneous RBS
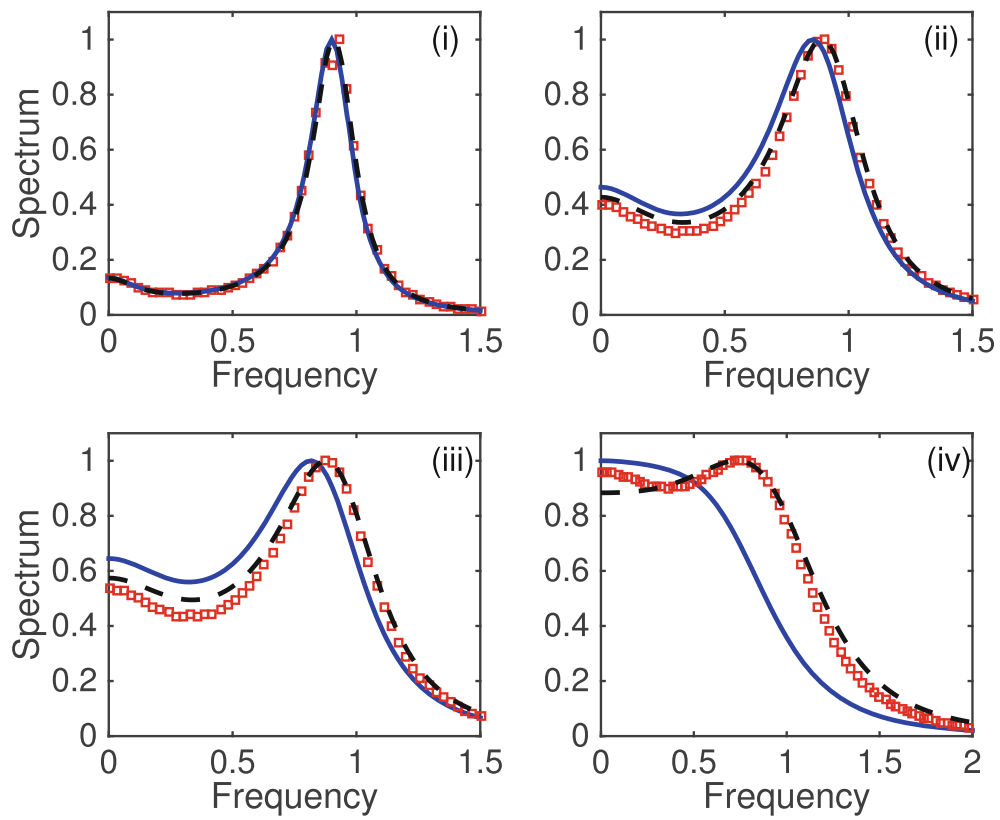

(b) Coherent RBS

Fig. 3 Spectra of the spontaneous and coherent RBS, when (i) $K n=0.02$, (ii) $K n=0.04$, (iii) $K n=0.05$, and (iv) $K n=0.1$. Solid and dashed lines are results from NS and Burnett equations, respectively. In this and subsequent figures, squares represent results from the LBE for Maxwellian gases if without specification

$-157 / 16$ that are derived to the third-order of $K n$ in the Chapman-Enskog expansion, perform much worse than the Burnett equations that are obtained from the ChapmanEnskog expansion to the second-order of $K n$, especially when $K n=0.1$. This conforms the 

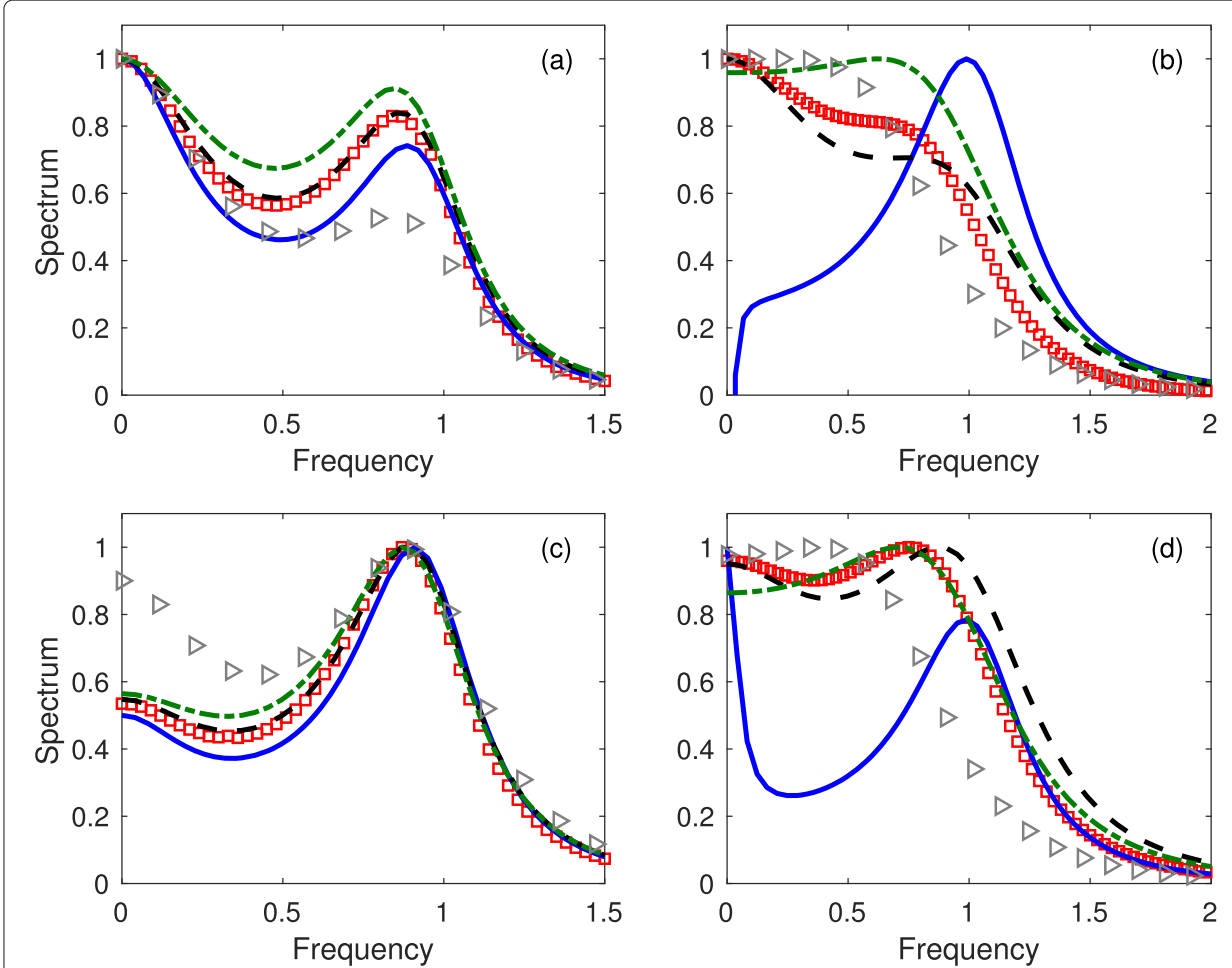

Fig. 4 Spectra of the spontaneous $(\mathbf{a}, \mathbf{b})$ and coherent $(\mathbf{c}, \mathbf{d}) \mathrm{RBS}$, when $(\mathbf{a}, \mathbf{c}) K n=0.05$ and $(\mathbf{b}, \mathbf{d}) K n=0.1$. Solid, dashed, and dash-dotted lines are the results from super-Burnett equations with $\theta_{7}=-157 / 16,-15 / 4$ and $11 / 16$, respectively. Triangles are results from the Woods' equations

criticism that one does not know what step of the approximation is required or sufficient to obtain a solution that is correct up to order $K n^{n}[9,63]$.

The augmented Burnett equations with $\theta_{7}=11 / 16$ do not do better. Interestingly, we observe in Fig. 4a and $\mathrm{c}$ that when $K n=0.05$, the RBS spectra from the LBE lie between those from the original and augmented Burnett equations. This inspires us to choose a different value of $\theta_{7}$ between $-157 / 16$ and $11 / 16$ to give a better agreement with the LBE solutions; eventually we find $\theta_{7}=-15 / 4$ works well in both spontaneous and coherent RBS. However, our stability analysis reveals that the augmented Burnett equation with $\theta_{7}=-15 / 4$ is not stable.

\subsection{Woods' modification at the Burnett level}

Woods argued that the Burnett equations are derived from an inappropriate framedependent formulation due to the improperly distinguishing between convection and diffusion [76]. A new approach to kinetic theory has been developed and the shear stress and heat flux at the Burnett level in dynamic light scattering are given by [52]:

$$
\sigma^{(W)}=\sigma^{(N S)}+2 K n^{2} \frac{\partial^{2} T}{\partial x^{2}}, \quad q^{(W)}=q^{(N S)}-\frac{15}{2} K n^{2} \frac{\partial^{2} u}{\partial x^{2}},
$$

which leads to the following analytical expressions for the RBS spectra: 


$$
\begin{array}{r}
S_{s}^{(W)}=\Re\left(\frac{i\left(1920 K n^{4} \pi^{6}-336 K n^{2} \pi^{4}+46 i K n \varpi \pi^{2}+3 \varpi^{2}-8 \pi^{2}\right)}{D^{(W)}}\right), \\
S_{c}^{(W)}=\left|\frac{6 \pi\left(10 \pi^{2} K n-i \varpi\right)}{D^{(W)}}\right|^{2},
\end{array}
$$

with $D^{(W)}=1920 K n^{4} \varpi \pi^{6}-336 K n^{2} \varpi \pi^{4}+46 i K n \varpi^{2} \pi^{2}-120 i K n \pi^{4}+3 \varpi^{3}-20 \varpi \pi^{2}$.

Some typical profiles of the RBS spectra are shown in Fig. 4. Compared to solutions of the Burnett equations in Fig. 3, we find that the performance of Burnett equations is better than Woods'.

We also note that another form of Burnett equations has also been proposed recently, based on Onsager's reciprocity principle, where the VDF is expanded as the function of thermodynamics forces and their corresponding fluxes [62]. However, in the linearized case, they are reduced to the linearized NS equations, and hence cannot describe the RBS spectra in the kinetic regime.

\subsection{Bi-velocity hydrodynamic equations}

The bi-velocity equations are derived based on the argument of "volume diffusion" $[7,8]$. In the study of normal shock wave structures, Greenshields and Reese found that the bi-velocity equations deliver an excellent match in the case of the inverse density thickness when compared to experimental data, by tuning the value of volume diffusivity [24]. However, in the problem of sound propagation in gases, Marques Jr. pointed out that Brenner's modification of continuum theory is unable to describe the acoustic measurements, not even in the low-frequency limit [40]. In the planar force-driven Poiseuille flow, Guo and $\mathrm{Xu}$ found that the Brenner model can yield some improved predictions when compared to the classical NS model, especially in the pressure field. However, it failed to give a qualitatively correct temperature profile. They hence concluded that "the Brenner's hydrodynamic model is still not adequate for describing gaseous micro flows" [31]. In the same problem, Dadzie and Brenner found that Brenner equations yield excellent agreement with the experimental mass flow rate up to a Knudsen number of 5 [14]. However, whether this good agreement comes from the boundary condition or the macroscopic equations is not clear.

The spectrum of spontaneous RBS has also been calculated based on Brenner's equations [3], though the accuracy has not been validated. Here we assess the performance of Brenner's equations in the dynamic light scattering, where the linearized shear stress and heat flux in Eq. (20) are given by

$$
\sigma^{(B N S)}=\sigma^{(N S)}-\frac{4 \alpha^{*}}{3} K n^{2} \frac{\partial^{2} \rho}{\partial x^{2}}, \quad q^{(B N S)}=q^{(N S)}-\alpha^{*} K n \frac{\partial \rho}{\partial x} .
$$

Brenner chose the volume diffusivity $\alpha_{v}$ in Eq. (8) to be the thermal diffusivity and hence $\alpha^{*}=1 / \operatorname{Pr}$, where $\operatorname{Pr}$ is the Prandtl number [7, 8]. However, Greenshields and Reese found that this choice would produce some unphysical results in the structures of normal shock waves. Instead, they suggested that $\alpha_{v}$ should take the value of the kinematic viscosity, that is, $\alpha^{*}=1[24]$.

Similar idea that the fluid velocity is composed of convective and diffusion velocities has been adopted to develop the extended NS equations $[16,55]$. In this light scattering problem, the shear stress in the linearized form remains unchanged, while the continuity equation, i.e. the first equation in Eq. (20), becomes 


$$
\frac{\partial \rho}{\partial t}+\frac{\partial u}{\partial x}=K n\left(\frac{\partial^{2} \rho}{\partial x^{2}}+\frac{1}{2} \frac{\partial^{2} T}{\partial x^{2}}\right),
$$

and the heat flux becomes

$$
q=-\frac{15}{4} K n \frac{\partial T}{\partial x}-\frac{3}{2} K n\left(\frac{\partial \rho}{\partial x}+\frac{1}{2} \frac{\partial T}{\partial x}\right)
$$

Typical profiles of the RBS spectra are shown in Fig. 5. When the Knudsen number is small, the gas dynamics falls in the continuum flow regime, it is surprising to find that the Brenner's and extended NS equations cannot even predict the RBS spectra, see Fig. 5a where there exists huge difference around the Brillouin peak. This is expected, for example, the effective heat conductivity is even changed in extended NS equations. In Brenner's model, the smaller value of $\alpha^{*}$ gives better results, because as $\alpha^{*}$ approaches zero, the model reduces to NS equations, which at least give the correct RBS spectra in the continuum flow regime.

\section{4 "Thermo-mechanically consistent" Burnett equations}

Probably inspired by Brenner's concept of "volume diffusion", Dadzie et al. proposed a Boltzmann-like gas kinetic equation and later derived the "thermo-mechanically consistent" Burnett equations [15]. In addition to the five basic hydrodynamical variables in NS equations, a scalar moment $\rho^{\prime}$ that has the dimension of a mass density is introduced [13].
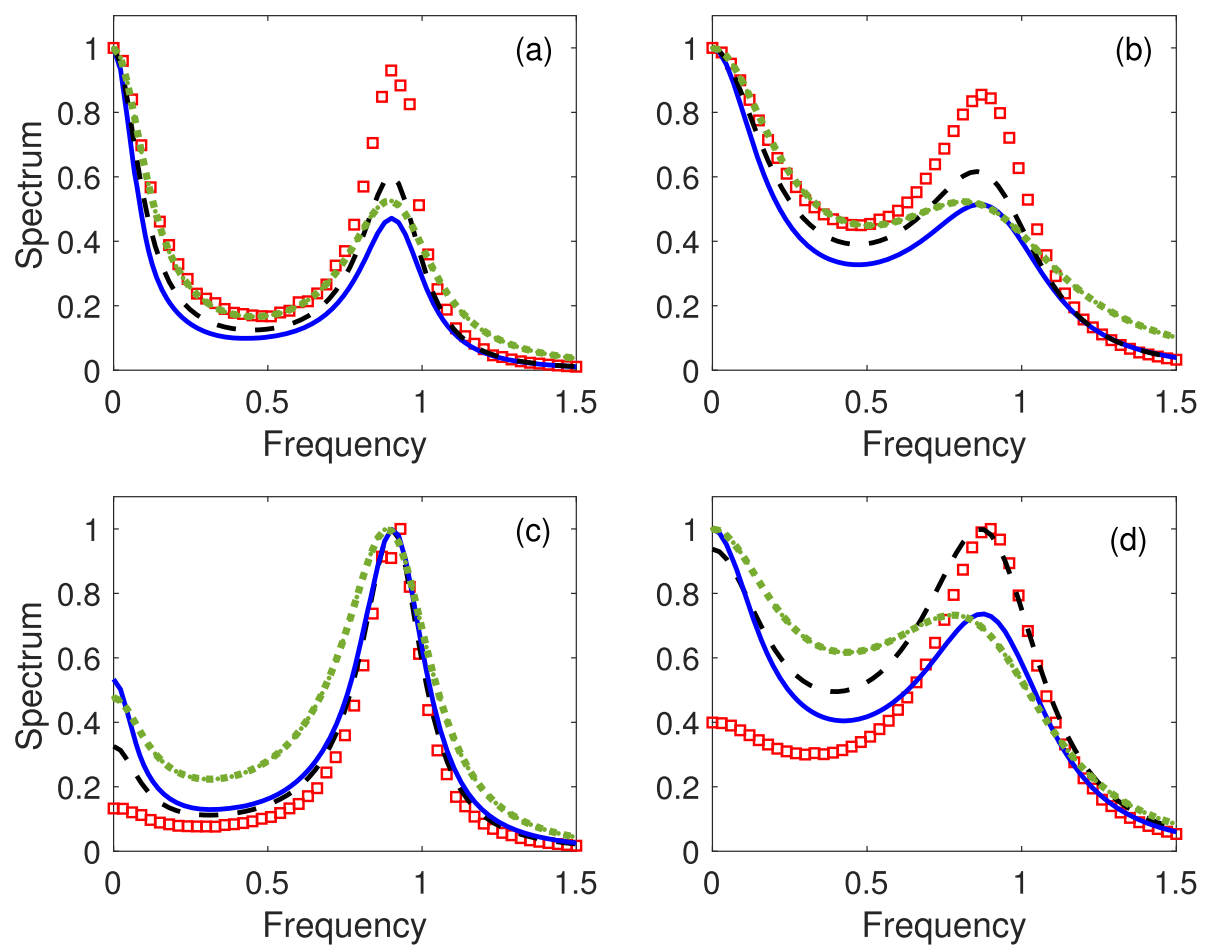

Fig. 5 Spectra of the spontaneous ( $a, b)$ and coherent $(c, d) R B S$, when $(a, c) K n=0.02$ and $(b, d) K n=0.04$. Solid and dashed lines are the results from Brenner's bi-velocity equations with $a^{*}$ in Eq. 30 being 3/2 and 1, respectively, while the dotted lines are results from the extended NS equations [55] 
The equation in the dimensionless form for the dynamic light scattering reads:

$$
\begin{aligned}
\frac{\partial \rho}{\partial t}+\frac{\partial u}{\partial x} & =0, \\
\frac{\partial \rho^{\prime}}{\partial t}-\frac{\partial \rho}{\partial t}-K n \kappa_{m} \frac{\partial^{2} \rho^{\prime}}{\partial x^{2}} & =0, \\
\frac{\partial u}{\partial t}+\frac{\partial \rho}{\partial x}+\frac{\partial T}{\partial x}-\frac{4 K n}{3} \frac{\partial^{2} u}{\partial x^{2}}-\frac{4 K n^{2}}{3} \kappa_{m} \frac{\partial^{3} \rho^{\prime}}{\partial x^{3}} & =a, \\
\frac{3}{2} \frac{\partial T}{\partial t}-\frac{\partial \rho^{\prime}}{\partial t}-K n \kappa \frac{\partial^{2} T}{\partial x^{2}} & =0
\end{aligned}
$$

where $\kappa_{m}=1 / \operatorname{Pr}$ and $\kappa=\gamma /(\gamma-1) \operatorname{Pr}$.

Taking the Prandtl number $\operatorname{Pr}=2 / 3$ and heat capacity ratio $\gamma=5 / 3$ for monatomic gases, we obtain $\kappa=15 / 4$. Hence Eq. (33) is reduced to the NS equations provided that only the zeroth- and first order terms of $K n$ are retained. Furthermore, it is found that the shear stress in Dadzie's model is exactly the same as that in Brenner's model. However, in a recent paper the parameter $\kappa_{m}$ is changed to $\operatorname{Pr} / 3$ [12].

Since $\rho^{\prime}$ does not have a clear physical meaning, we do not know whether the initial density impulse has an effect on the Laplace-Fourier transform of $\rho^{\prime}$ or not: remember in spontaneous RBS the Laplace-Fourier transform of the term $\partial \rho / \partial t$ is $-i \varpi \hat{\rho}-1$ due to the initial density impulse; however, we do not know what is the Laplace-Fourier transform of the term $\partial \rho^{\prime} / \partial t$ is. Therefore, we only consider the coherent RBS, where the Fourier transform of both $\partial \rho / \partial t$ and $\partial \rho^{\prime} / \partial t$ are zero. The spectrum of coherent RBS can be obtained by solving the following matrix:

$$
\left[\begin{array}{cccc}
-i \varpi & 2 i \pi & 0 & 0 \\
2 i \pi & -i \varpi+\frac{16}{3} \pi^{2} K n & 2 i \pi & \frac{32}{3} i \pi^{3} K n^{2} \kappa_{m} \\
0 & 0 & -\frac{3}{2} i \varpi+4 \pi^{2} K n \kappa & i \varpi \\
i \varpi & 0 & 0 & -i \varpi+4 \pi^{2} K n \kappa_{m}
\end{array}\right]\left[\begin{array}{c}
\hat{\rho} \\
\hat{u} \\
\hat{T} \\
\hat{\rho^{\prime}}
\end{array}\right]=\left[\begin{array}{l}
0 \\
1 \\
0 \\
0
\end{array}\right]
$$

with $\hat{\rho}^{\prime}$ being the spatial-temporal Fourier transform of $\rho^{\prime}$.

Typical profiles of the coherent RBS spectra are depicted in Fig. 6. It is surprising to note that, when $\kappa_{m}=1 / P r$, Dadzie's model cannot even predict the spectrum in the regime where NS equations are valid, say, when $K n=0.02$ : the position of the Rayleigh peak is shifted away from zero frequency. This problem becomes more severer when the Knudsen number increases, where a strong deviation from the LBE solution is seen in Fig. $6 \mathrm{~b}$ even when $K n=0.04$. The drift of center of Rayleigh peak is induced by the second equation in Eq. (33). When $\kappa_{m}$ is changed to $\operatorname{Pr} / 3$, this problem is alleviated and good agreement is achieved when $K n=0.02$, but for larger Knudsen numbers, Dadzie's model is still not good enough to yield reasonable spectrum of the coherent RBS. One may conjecture that a further reduction of $\kappa_{m}$ may lead to excellent agreement with the LBE solution at, say $K n=0.04$, but our numerical results show that although the spectrum near the Rayleigh peak agrees better, the spectrum near the Brillouin peak shifts leftwards, hence the overall line shape is even worse. 

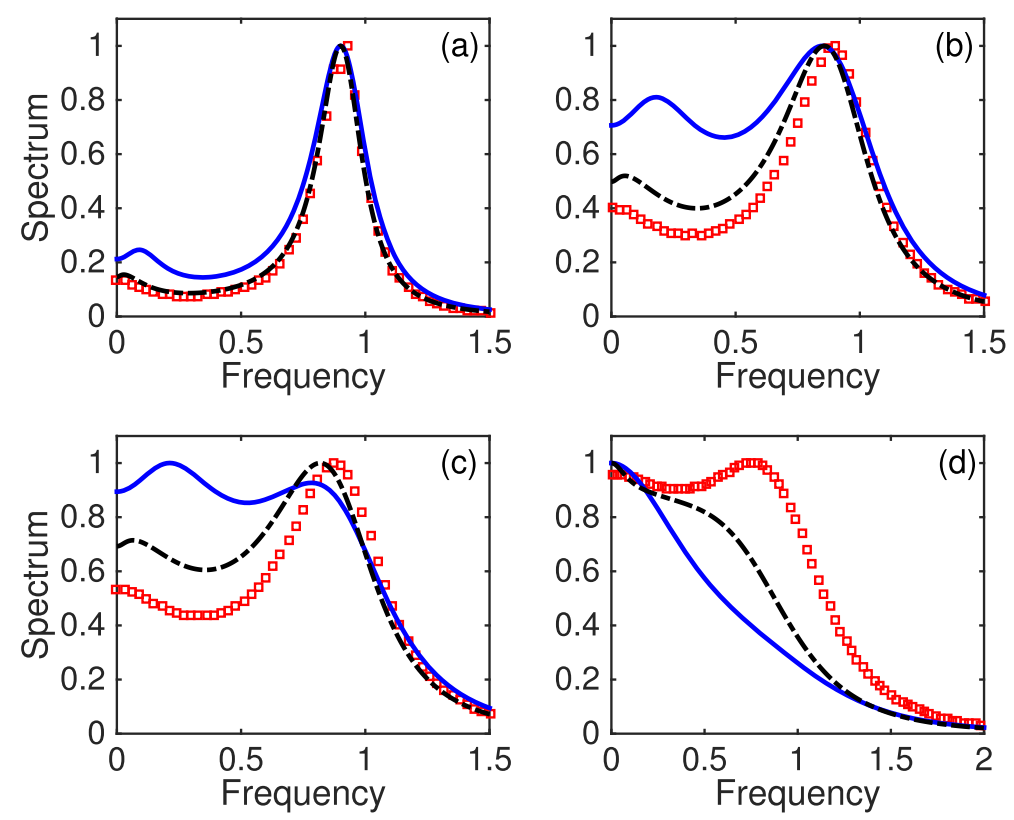

Fig. 6 Spectra of the coherent RBS, when (a) $K n=0.02$, (b) $K n=0.04$, (c) $K n=0.05$, and (d) $K n=0.1$. Solid and dashed lines are results from Dadzie's equations (33) with $\kappa_{m}=3 / 2$ and $\kappa_{m}=2 / 9$, respectively

\subsection{Moment equations}

Moment equations are derived through the method of ansatz, that is, the $\operatorname{VDF} f(t, \mathbf{x}, \mathbf{v})$ in the Boltzmann equation (3) is assumed to be the product of Gaussian function and the sum of several low-order Hermite polynomials with the coefficients related to the moments of VDF. The first set of moment equations are obtained by Grad consisting of 13 macroscopic quantities [23], where the VDF is given by Eq. (7). In this dynamic light scattering problem the shear stress and heat flux in the G13 equations satisfy the following equations:

$$
\begin{aligned}
& \frac{\partial \sigma}{\partial t}+\frac{4}{3} \frac{\partial u}{\partial x}+\frac{8}{15} \frac{\partial q}{\partial x}=-\frac{\sigma}{K n} \\
& \frac{\partial q}{\partial t}+\frac{\partial \sigma}{\partial x}+\frac{5}{2} \frac{\partial T}{\partial x}=-\frac{2}{3} \frac{q}{K n}
\end{aligned}
$$

The spectra of spontaneous and coherent RBS can be obtained by solving the following matrix:

$$
\left[\begin{array}{ccccc}
-i \varpi & 2 i \pi & 0 & 0 & 0 \\
2 i \pi & -i \varpi & 2 i \pi & 2 i \pi & 0 \\
0 & 2 i \pi & -\frac{3}{2} i \varpi & 0 & 2 i \pi \\
0 & \frac{8}{3} i \pi & 0 & -i \varpi+\frac{1}{K n} & \frac{16}{15} i \pi \\
0 & 0 & 5 i \varpi & 2 i \varpi & -i \varpi+\frac{2}{3 K n}
\end{array}\right]\left[\begin{array}{c}
\hat{\rho} \\
\hat{u} \\
\hat{T} \\
\hat{\sigma} \\
\hat{q}
\end{array}\right]=\left[\begin{array}{c}
N_{1} \\
N_{2} \\
0 \\
0 \\
0
\end{array}\right],
$$

where in the coherent RBS $\hat{\sigma}$ and $\hat{q}$ are respectively the spatial-temporal Fourier transform of $\sigma$ and $q$, while in spontaneous RBS $\hat{\sigma}$ and $\hat{q}$ are respectively the Laplace-Fourier transform of $\sigma$ and $q$ in the temporal-spatial directions. 
Note that G13 equations are accurate to the second-order of $K n$, which have been extended to R13 equations that are accurate to the third-order of $K n$ [37, 64]. The governing equations for the shear stress and heat flux, as according to Struchtrup [64], are

$$
\begin{aligned}
& \frac{\partial \sigma}{\partial t}+\frac{4}{3} \frac{\partial u}{\partial x}+\frac{8}{15} \frac{\partial q}{\partial x}-\frac{6}{5} K n \frac{\partial^{2} \sigma}{\partial x^{2}}=-\frac{\sigma}{K n}, \\
& \frac{\partial q}{\partial t}+\frac{\partial \sigma}{\partial x}+\frac{5}{2} \frac{\partial T}{\partial x}-\frac{18}{5} K n \frac{\partial^{2} q}{\partial x^{2}}=-\frac{2}{3} \frac{q}{K n} .
\end{aligned}
$$

Similarly, the evolution of the shear stress and heat flux in the linearized R26 equations, which are accurate up to the fifth-order of $K n$, are given by $\mathrm{Gu}$ and Emerson as [27]:

$$
\begin{array}{r}
\frac{\partial \sigma}{\partial t}+\frac{4}{3} \frac{\partial u}{\partial x}+\frac{8}{15} \frac{\partial q}{\partial x}+\frac{\partial \bar{m}}{\partial x}=-\frac{\sigma}{K n} \\
\frac{\partial q}{\partial t}+\frac{5}{2} \frac{\partial T}{\partial x}+\frac{\partial \sigma}{\partial x}+\frac{1}{6} \frac{\partial \bar{\Delta}}{\partial x}+\frac{1}{2} \frac{\partial \bar{R}}{\partial x}=-\frac{2}{3} \frac{q}{K n}
\end{array}
$$

where the higher-order moments $\bar{m}, \bar{\Delta}$, and $\bar{R}$ are governed by the following equations:

$$
\begin{array}{r}
\frac{\partial \bar{m}}{\partial t}+\frac{9}{5} \frac{\partial \sigma}{\partial x}+\frac{9}{35} \frac{\partial \bar{R}}{\partial x}-\frac{16}{7} \frac{K n}{A_{\phi 1}} \frac{\partial^{2} \bar{m}}{\partial x^{2}}=-A_{m} \frac{\bar{m}}{K n} \\
\frac{\partial \bar{\Delta}}{\partial t}+8 \frac{\partial q}{\partial x}-\frac{7 K n}{3} \frac{\partial^{2} \bar{\Delta}}{\partial x^{2}}-\frac{4 K n}{A_{\Omega 1}} \frac{\partial^{2} \bar{R}}{\partial x^{2}}=-A_{\Delta 1} \frac{\bar{\Delta}}{K n} \\
\frac{\partial \bar{R}}{\partial t}+\frac{56}{15} \frac{\partial q}{\partial x}+2 \frac{\partial \bar{m}}{\partial x}-\frac{K n}{5}\left(\frac{54}{7 A_{\psi 1}}+\frac{16}{3 A_{\Omega 1}}\right) \frac{\partial^{2} \bar{R}}{\partial x^{2}}-\frac{28 K n}{45 A_{\Omega 1}} \frac{\partial^{2} \bar{\Delta}}{\partial x^{2}}=-A_{R 1} \frac{\bar{R}}{K n}
\end{array}
$$

with for Maxwellian molecules we have $A_{m}=1.5, A_{\Delta 1}=2 / 3, A_{\Omega 1}=1, A_{\phi 1}=2.097$, $A_{\psi 1}=1.698$, and $A_{R 1}=7 / 6$.

Figure 7 shows the spectra of both instantaneous and coherent RBS obtained from the G13, R13, and R26 moment equations. When $K n=0.02$, all the moment equations predict the same spectrum as that from the LBE. However, even when $K n$ is increased to $K n=0.04$, spectra from the G13 equations deviate significantly from those of the LBE. The $\mathrm{R} 13$ equations are accurate up to $K n \approx 0.04$, while the R26 equations are accurate up to $K n \approx 0.06$.

We have also derived the regularized 35 moment equations, where the distribution function is expanded to the fifth-order polynomials and all the relevant moments are included. For the dynamic light scattering problem, only one extra equation for a higher-order moment is added:

$$
\frac{\partial \bar{\phi}}{\partial t}+\frac{16}{7} \frac{\partial \bar{m}}{\partial x}-\frac{96 K n}{245 A_{\psi 1}} \frac{\partial^{2} \bar{R}}{\partial x^{2}}-\frac{25 K n}{9 A_{w 1}} \frac{\partial^{2} \bar{\phi}}{\partial x^{2}}=-\frac{A_{\phi 1}}{K n} \bar{\phi},
$$

with $A_{w 1}=2.743$ for Maxwellian molecules, while the equation for $\bar{m}$ in Eq. (39) is replaced by

$$
\frac{\partial \bar{m}}{\partial t}+\frac{9}{5} \frac{\partial \sigma}{\partial x}+\frac{9}{35} \frac{\partial \bar{R}}{\partial x}+\frac{\partial \bar{\phi}}{\partial x}=-\frac{3}{2} \frac{\bar{m}}{K n} .
$$

We have noted that from 1D shear flow R35 equations are better than R26 equations, but not much. The same conclusion can be made in the dynamic light scattering problem, where the spectra from R26 and R35 equations are nearly identical, since there are both 

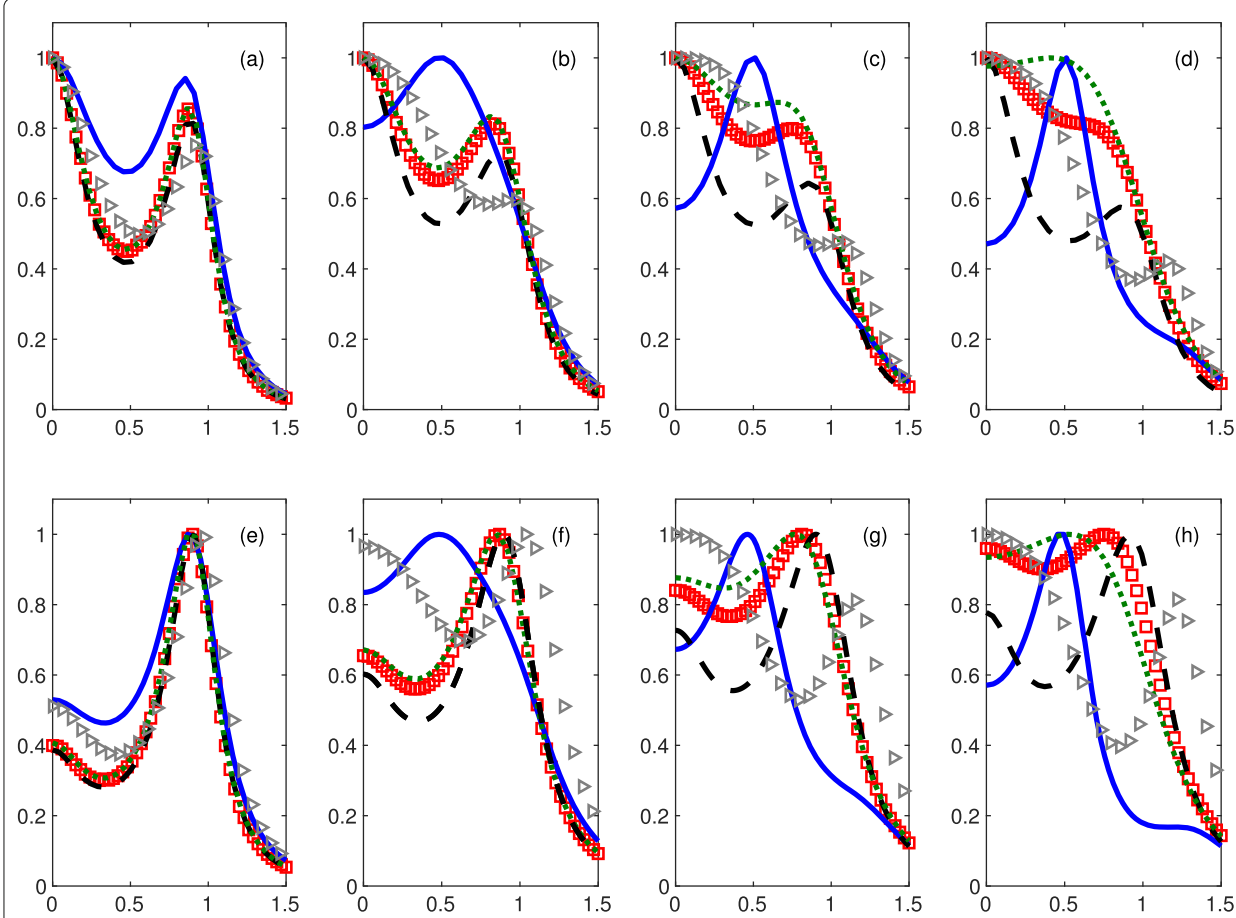

Fig. 7 Spectra of the spontaneous (top row) and coherent (bottom row) RBS. The horizontal and vertical axis are the normalized frequency and spectrum, respectively. Form the left to right, the Knudsen number in each column are $0.04,0.06,0.08$, and 0.1 , respectively. Solid, dashed, and dotted lines are the results from G13, R13, and R26 moment equations, respectively, while triangles are results from Eu's generalized hydrodynamic equations

accurate to $O\left(K n^{5}\right)$. In 2D or 3D situations, the computational costs increase as more equations are included in the system, but the accuracy is not gained proportionally.

\subsection{Generalized hydrodynamic equations}

Eu also developed a new set of macroscopic equations called the generalized hydrodynamic equations, by constructing a non-equilibrium distribution function that ensures the non-negativity property of entropy production [18]. Unlike the moment method where the distribution function is the product of Gaussian function and Hermite polynomials, the distribution function in Eu's method is an exponential function of some low-order Hermite polynomials. In the dynamic light scattering by rarefied gases, the evolution of the shear stress and heat flux are governed by

$$
\begin{gathered}
\frac{\partial \sigma}{\partial t}+\frac{4}{3} \frac{\partial u}{\partial x}=-\frac{\sigma}{K n}, \\
\frac{\partial q}{\partial t}+\frac{5}{2} \frac{\partial T}{\partial x}=-\frac{2}{3} \frac{q}{K n},
\end{gathered}
$$

which, due to the decoupling between the shear stress and heat flux, are simpler than those in G13 equations (35).

The RBS spectra are shown in Fig. 7 at several typical Knudsen numbers. It is seen that the accuracy of Eu's model lies between that of G13 and R13 moment equations, which is worse than the Burnett and Brenner equations. 
Note that for nonlinear gas flows, the constitutive relations in Eu's original equations are coupled and cannot be solved easily. Based on the adiabatic assumption and simplifications made by Myong [46, 47], nonlinear coupled constitutive relations have been developed and applied to hypersonic flows. In some cases, very good agreement with results from the direct simulation Monte Carlo method [4] is observed [82], even at relative large Knudsen numbers. However, in the linearized case, the nonlinear coupled constitutive relations are reduced to those in the linearized NS equations. Therefore, in the spontaneous and coherent RBS it works only in the hydrodynamic regime.

Very recently Rana et al. also proposed the coupled constitutive relations by combining ideas of different approaches to irreversible thermodynamics, in particular the rational (extended) thermodynamics [51]. In RBS, linearized expressions for the shear stress and heat flux are

$$
\begin{gathered}
\sigma=\sigma^{(N S)}-\frac{8}{15} K n \frac{\partial q}{\partial x} \\
q=q^{(N S)}-\frac{3}{2} K n \frac{\partial \sigma}{\partial x} .
\end{gathered}
$$

Numerical results for the spectra of both spontaneous and coherent RBS, however, suggest that the coupled constitutive relations have similar accuracy as that of the G13 equations (not shown here).

\subsection{Rational extended thermodynamics}

Rational extended thermodynamic equations for rarefied gas dynamics are derived from the gas kinetic equation in a manner similar to the derivation of moment equations, but the distribution function is instead obtained by the maximum entropy principle. Recently, it has been used to study the spontaneous RBS spectra in polyatomic gases by considering only 14 moments (i.e. in addition to the 13 moments in G13 equations, the dynamical pressure that is related to the bulk viscosity is also considered); the corresponding macroscopic equations can be found in the Chapter 9 of the book [54], which degenerate to G13 equations for monatomic gases when the deviation from equilibrium is weak.

The spectrum of spontaneous RBS from the rational extended thermodynamics with 14 moments has been compared with the experiment [25], where the light with the effective wave vector $k=1.98 \times 10^{5} \mathrm{~cm}^{-1}$ is scattered by $\mathrm{CO}_{2}$ at a temperature $298 \mathrm{~K}$ and pressure $750 \mathrm{~mm} \mathrm{Hg}$. In the numerical calculation of the spectrum of spontaneous RBS based on the LBE for polyatomic gas, we use the fast spectral method developed in Ref. [80], with the ratio of the bulk viscosity to the shear viscosity of $\mathrm{CO}_{2}$ being 0.39 [30]. From Fig. 8 we can find the huge difference between the results of rational extended thermodynamics and experiment, while our LBE solution gives a good prediction of the RBS spectrum. We conclude that the accuracy of the rational extended thermodynamics is roughly at the same order with G13 equations based on the essence in the derivation of these macroscopic equations.

\subsection{Discussions}

Based on benchmarking solutions from the LBE for the RBS spectra, we have assessed the accuracy of more than ten macroscopic equations. The results are summarized and visualized more clearly in Fig. 9, where the relative difference in the RBS spectra between solutions from macroscopic equations and LBE is shown as a function of the Knudsen 


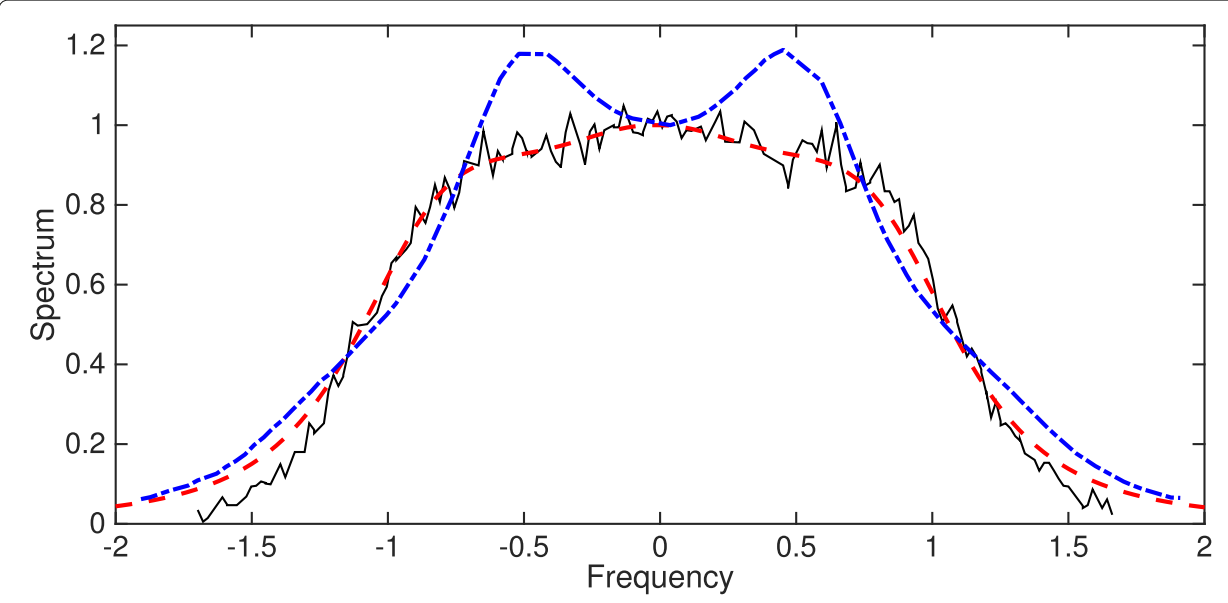

Fig. 8 Comparisons in the spectra of spontaneous RBS between the experimental data (the solid line) from Ref. [25], the LBE (the dashed line) for polyatomic gas [80], and the rational extended thermodynamics with 14 moments (the dash-dotted line) by Ruggeri and Sugiyama [54]. The light is scattered from $\mathrm{CO}_{2}$ when $K n=0.1114$. Note that the frequency is normalized by $1.06 \mathrm{GHz}$, and the shown spectra are the convolution of $S_{s}\left(K n, f_{s}\right)$ and the Lorentzian function $\operatorname{Lor}\left(f_{s}\right)$ with the Full Width at Half Maximum being $210 \mathrm{MHz}$, i.e., $\operatorname{Lor}\left(f_{s}\right) \propto 1 /\left(f_{s}^{2}+9.8 \times 10^{-3}\right)$

number. The solution may be viewed being accurate when the relative difference is less than 0.05. It should be noted that in dynamic light scattering both the spatial and temporal Knudsen numbers, as defined in Eqs. (10) and (15), play important roles. For steady-state problems, the range of applicability of these macroscopic equations may be expanded to larger values of $K n$.

Interestingly, as the order of Chapman-Enskog expansion increases, the accuracy of the obtained macroscopic equations does not necessarily increase (say, when $K n \gtrsim 0.03$, super-Burnett equations are less accurate than Burnett equations), which confirms the criticism that "one does not know what step of the approximation is required or sufficient to obtain a solution that is correct up to order $K n^{n}$. For the (regularized) moment equations, however, the accuracy in the prediction of RBS spectra is consistent with the accuracy in deriving these equations, that is, increases monotonically from G13, R13, to R26 moment equations. Eu's generalized hydrodynamic equations, where the VDF contains the fourth-order Hermite polynomial, has the same level accuracy as G13 equations, where the distribution function is expanded only up to the third-order Hermite polynomial, and it is less accurate than R13 equations. Brenner's bi-velocity fluid model and Dadzie's "thermomechanically consistent" Burnett equations, which contain free parameters that can not be determined from fundamental physical laws, both involve the concept of "volume diffusion", do not perform well compared to R13 equations, even when the best parameters are selected. This is because there is a lack of solid argument from actual microphysics in these methods. For example, just because the Burnett equations derived from the Boltzmann equation does not have good properties, the "thermo-mechanically consistent" Burnett equation was proposed by adding non-physical "volume diffusion" term to the Boltzmann equation $[13,15]$. This logic is not correct because that the Burnett equations are not good does not mean the Boltzmann equation is wrong. In fact since the Boltzmann equation was proposed there is no single evidence that it fails to describe the 

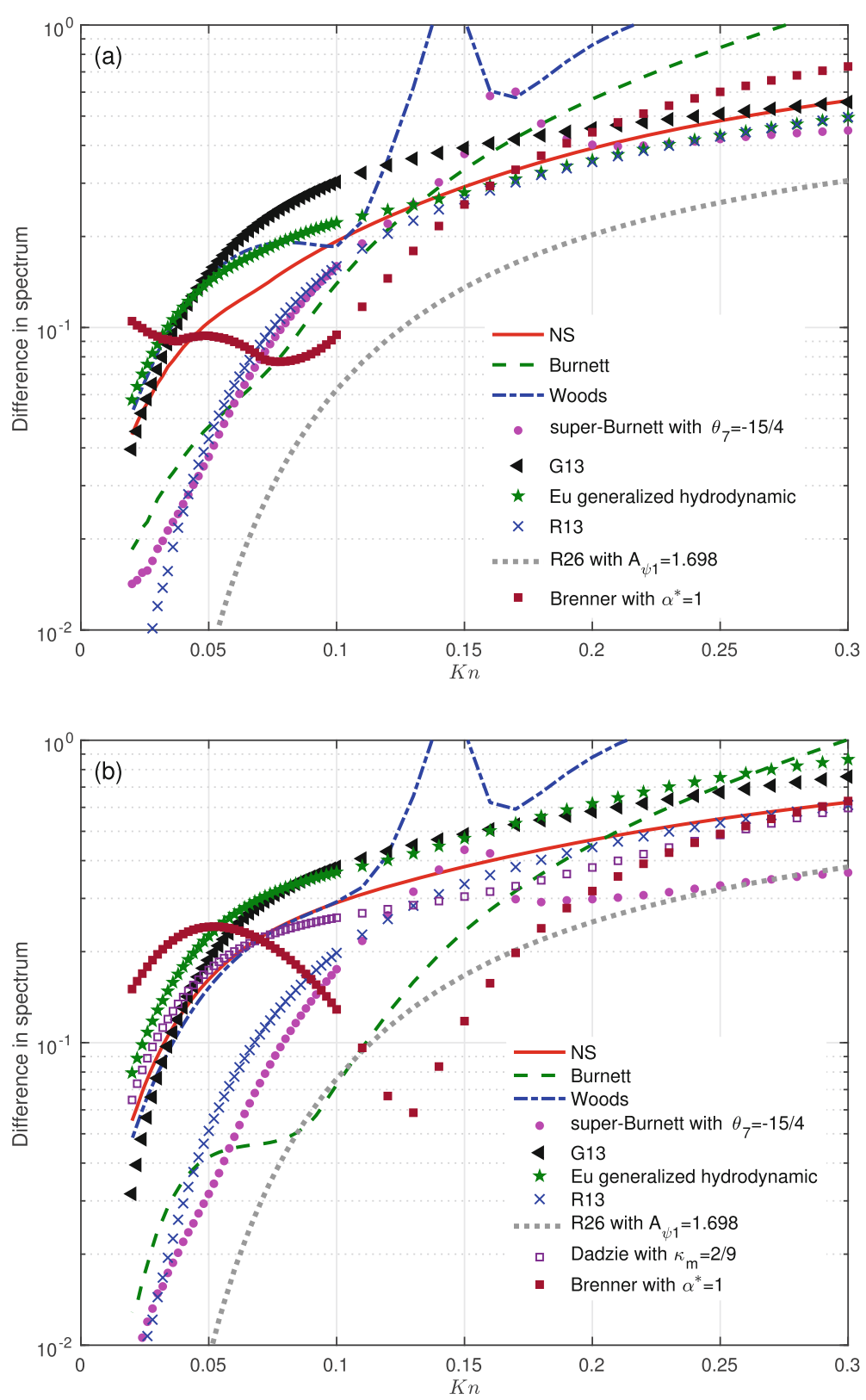

Fig. 9 Difference $\int_{\infty}^{\infty}\left|S^{L B E}-S^{M a c}\right| d f_{s}$ in the spectrum of spontaneous (a) and coherent (b) RBS between solutions of LBE and macroscopic equations. Note that before the comparison, areas of RBS spectra are normalized to unity. The solution may be viewed to be accurate when the relative difference is less than 0.05 . Note that super-Burnett with $\theta_{7}=-15 / 4$, Brenner's, and Dadzie's equations are purely phenomenological models with arbitrary tuned parameters to give their best results, while other macroscopic equations in this figure are derived without any parameter tuning

rarefied gas dynamics of dilute gases, while the "thermo-mechanically consistent" Burnett equations are often changed in order to fit the theory of Boltzmann equation as well as experimental data.

\subsubsection{Convergence of moment systems}

From these comparisons, it is concluded that the the moment method is a proper way to derive higher-order macroscopic equations to describe the rarefied gas dynamics: 
the derivation is straightforward, and its accuracy is definitive and controllable, that is, increases with the number of moments or order of Hermite polynomials used to approximate the VDF. This is quite important in numerical simulations, where the error may be analyzed in prior. To further illustrate this point, we study the performance of higher-order moment systems, by solving the linearized Shakhov gas kinetic model [57] numerically using the discrete velocity method based on the Gauss-Hermite quadrature, which is equivalent to the Grad moment method at different order of approximations; more detailed analysis is given in Ref. [58]. We also noted that a similar research has been done to find the minimum number of moments in rational extended thermodynamics to describe the light spectrum [74].

We choose the spontaneous RBS as an example. Under the same normalization as used in section 2, the evolution of the VDF for perturbation $h\left(t, x_{1}, \mathbf{v}\right)$ is governed by the following linearized Shakhov equation:

$$
\frac{\partial h}{\partial t}+v_{x} \frac{\partial h}{\partial x_{1}}=\delta\left[\rho+2 u_{1} v_{1}+T\left(|\mathbf{v}|^{2}-\frac{3}{2}\right)+\frac{4 q_{1} v_{x}}{15}\left(|\mathbf{v}|^{2}-\frac{5}{2}\right)-h\right]
$$

where $\rho=\int h \mathrm{~d} \mathbf{v}$ is the perturbed number density, $u_{1}=\int v_{1} h \mathrm{~d} \mathbf{v}$ is the perturbed velocity, $T=\frac{2}{3} \int|\mathbf{v}|^{2} h \mathrm{~d} \mathbf{v}-\rho$ is the perturbed temperature, $q_{1}=\int|\mathbf{v}|^{2} v_{1} h \mathrm{~d} \mathbf{v}-\frac{5}{2} u_{1}$ is the perturbed heat flux, and $\delta=1 / \sqrt{2} K n$. When the molecular velocity space $\mathbf{v}$ is discretized by GaussHermite quadrature, the spectrum can be obtained by using a similar iterative scheme as in Eq. (14). If the $N$-th order Gauss-Hermite quadrature is considered, the moment up to the order $\left\lfloor\frac{2 N-1}{2}\right\rfloor=N-1$ can be captured accurately [58]. Consider the fact that G13 equations where the VDF is expanded up to third-order Hermite polynomials are accurate to $O\left(\mathrm{Kn}^{2}\right)$, the numerical solution of the Shakhov equation based on the Gauss-Hermite quadrature of order $N$ has an accuracy of $O\left(\mathrm{Kn}^{\mathrm{N}-2}\right)$.

Using solutions of the Shakhov model approximated by the 60th-order Gauss-Hermite quadrature as reference, we analyze the accuracy of various orders of moment equations in Fig. 10. We also show the relative error in the spectrum from comparisons between G13/R13/R26 equations and the LBE. When $N=4$, the Gauss-Hermite quadrature yields an equivalent moment system accurate to $O\left(K n^{2}\right)$, therefore, the relative difference curve almost overlaps that from G13 equations. Similarly, $N=5$ and 7 yield equivalent moment systems accurate to $O\left(\mathrm{Kn}^{3}\right)$ and $O\left(\mathrm{Kn}^{5}\right)$, respectively. Therefore, relative difference curves nearly overlap with those from R13 and R26 moment equations in a wide range of Knudsen numbers. Obviously, as more moments (i.e. higher-order quadrature) are included, the accuracy increases monotonically.

However, it is seen from Fig. 10 that at large values of $K n$ the convergence rate of moment equations is slow. For example, when $K n=0.3$, when $N$ is increased from 5 to 20, that is, when the accuracy of the equivalent moment systems is increased from $O\left(K n^{3}\right)$ to $O\left(K n^{18}\right)$, the error is only reduced by about one order of magnitude. And the solution of $N=20$ can be marginally viewed as accurate when compared to the converged solution; and it is still not accurate enough to predict the RBS spectrum. Accuracy of moment systems may become worse in wall-bounded problems such as the Poiseuille flow between two parallel plates [68], as Gauss-Hermite polynomials are not good at capturing discontinuities in the VDF. The performance of moment systems of various orders will be investigated in the wall-bounded sound propagation problem in the next section. 


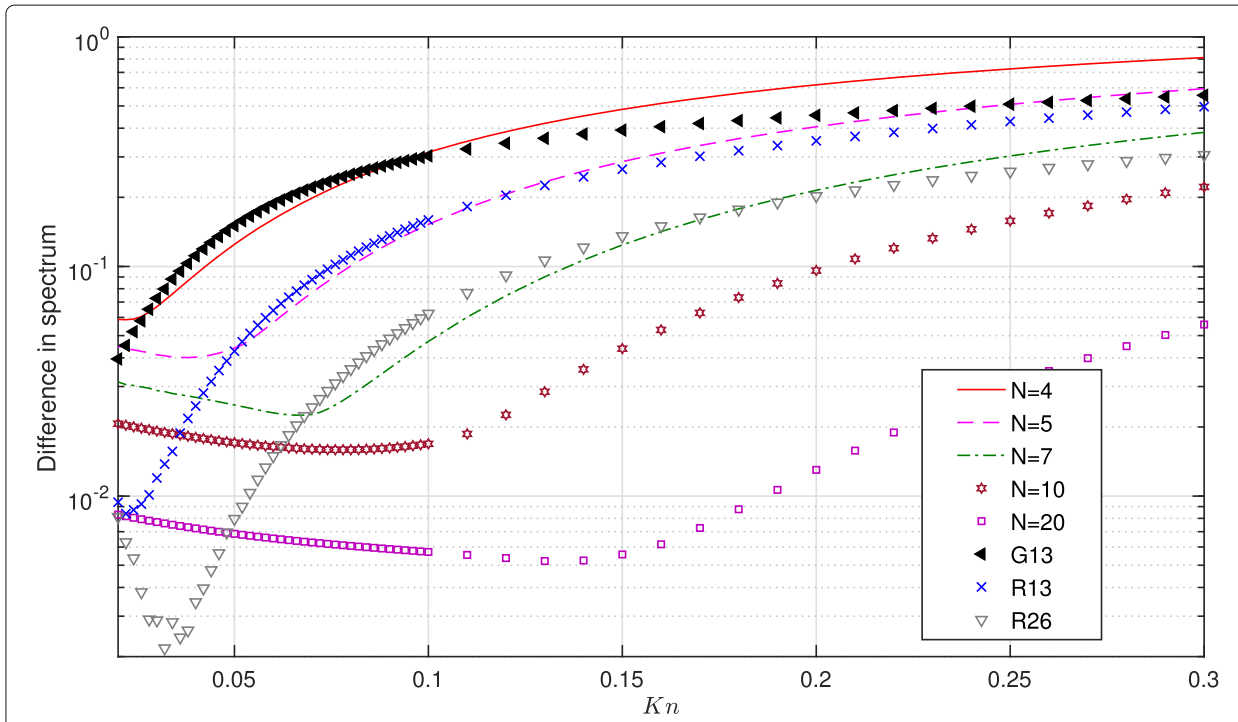

Fig. 10 Difference in the spectrum of spontaneous RBS. Results for G13, R13, and R26 moment equations are obtained from Fig. 9a, while others are $\int_{\infty}^{\infty}\left|S_{G H}^{N}-S_{G H}^{N=60}\right| d f_{s}$ with $S_{G H}^{N}$ being the spectrum obtained by solving the Shakhov kinetic model (44) with the Gauss-Hermite quadrature of order $N$; in three-dimensional molecular velocity space $\mathbf{v}$, the number of total discrete velocity points is $N^{3}$, while the corresponding Grad moment equations have $(N+1)(N+2)(N+3) / 6$ moments [19]. Note that before the comparison, areas of RBS spectra are normalized to unity. The solution may be viewed to be accurate when the relative difference is less than 0.05. Also note that the relative difference when $N=5,7,10$ and 20 does not decrease with $K n$ when $K n \lesssim 0.1$ is probably because in spontaneous RBS the spectrum between the Rayleigh and Brillouin peaks is nearly zero [see Fig. 2a] so any difference is magnified

\section{Sound propagation between the transducer and receiver}

We now test the accuracy of macroscopic equations in the problem of sound propagation in gas confined between the transducer and receiver. As shown in Fig. 11, a monatomic gas is enclosed between two walls with a distance of $L$, measured along the $x$-coordinate. The left wall is the transducer which oscillates periodically with the velocity

$$
u=u_{0} \cos (\varpi t)
$$

while the other wall is the resting receiver where the gas pressure is recorded. The velocity amplitude $u_{0}$ is so small that the gas is only slightly disturbed, therefore it is sufficient

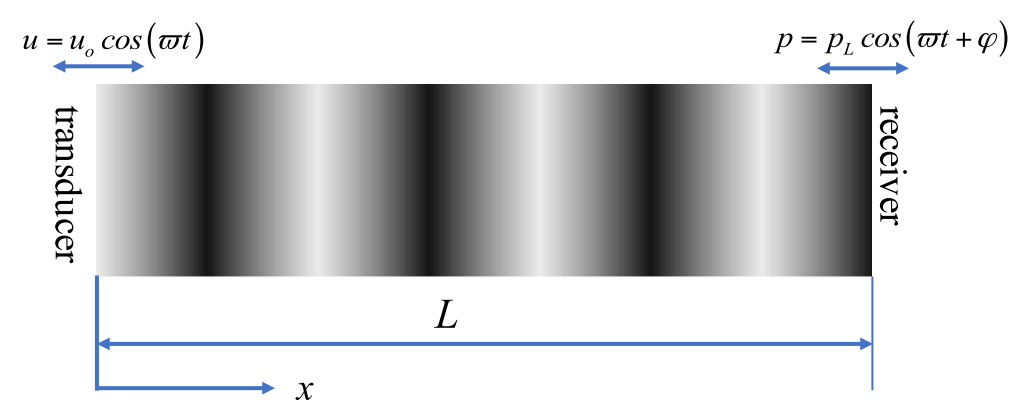

Fig. 11 Schematic of the sound propagation between transducer and receiver. The sound is generated by the periodic oscillation of the transducer, while the receiver measures the pressure amplitude and the phase shift. The dimensions perpendicular to the $x$-direction are much bigger than $L$, so that the problem is 1D 
to consider all transport equations in linearized form. The quantity of interest is the normal pressure exerted on resting receiver in the $x$-direction, which also oscillates with the frequency $\varpi$ but with a phase shift $\phi$. That is, its expression is given by

$$
p(t)=\rho+T+\sigma \equiv p_{L} \cos (\varpi t+\phi) .
$$

We use the gas kinetic equation (44) to describe the rarefied gas dynamics, as (i) the previous study showed that this model equation can provide accurate results when compared to that of LBE, with relative error in pressure less than 4\% [77], and (ii) the GaussHermite quadrature can be applied to this equation [58] to mimic the behavior of moment equations at any order, to replace the complicated derivation and solving of high-order moment equations beyond R26. We use Maxwell's diffuse boundary condition to describe the gas-wall interaction [43]; detailed form of which can be found in Ref. [77] and the efficient numerical method to solve Eq. (44) is given in Ref. [69].

Macroscopic equations are the same as in the dynamic light scattering problem, except for R26 equations we have $A_{m}=A_{\Delta 1}=A_{\Omega 1}=A_{\phi 1}=A_{\psi 1}=A_{R 1}=1$ in Eq. (39), as derived from the Shakhov kinetic model (44). The gas-surface interaction adds additional difficulty. That is, boundary conditions for high-order equations like Burnett, super-Burnett, Woods, "thermo-mechanically consistent" Burnett, and generalized hydrodynamic equations are hard to construct; hence they will not be considered here. Brenner's and extended NS equations will not be considered either since they are not accurate even in the near-continuum flow regime. Neither will we consider G13 and rational extended thermodynamics equations, since they are equivalent in this problem, and it has already been shown that they are not as accurate as R13 equations [66]. This leaves us to only the NS, R13, and R26 equations. The boundary conditions for NS and R13 equations corresponding to the diffuse boundary condition for gas kinetic model equations can be found in Ref. [66], while that for R26 equations is given by Ref. [27].

We first compare solutions of the Shakhov model (44) with the experimental data of Ref. [56]. Although his results suggested that "the accommodation at the transducer surfaces must be incomplete", comparisons in Fig. 12 show that the diffuse boundary condition can predict the normal pressure at the receiver, except in the region where $\sqrt{\pi} \varpi L / \nu_{m}$ is small, see Fig. 12a and d. This is actually consistent with the discovery in Ref. [36] that in some parameter regions the pressure is sensitive to gas-surface boundary condition while in other regions it is not. Therefore, in the following, the accuracy of macroscopic equations will be assessed only by comparing with solutions of the kinetic model (44) using the equivalent diffuse boundary condition.

Note that the parameter $\varpi \tau_{c}$ in Fig. 12 is proportional to the Knudsen number defined in the time domain $K n_{t}$ as $\varpi \tau_{c}=2 \sqrt{2} \pi K n_{t}$, see Eq. (15). Therefore, as $\varpi \tau_{c}$ increases, the rarefaction effects become more and more strong, so macroscopic equations gradually lose accuracy. The NS equations are accurate when $\varpi \tau_{c}=0.1$ (not shown), but are already very inaccurate when $\varpi \tau_{c}=0.3$, see Fig. 12a. R13 equations, which are accurate to the order of $\mathrm{Kn}^{3}$, give reasonable good results up to $\varpi \tau_{c}=0.3$, see Fig. $12 \mathrm{~b}$. While R26 equations, which are accurate to the order of $K n^{5}$, predict the normal pressure at the receiver fairly well up to $\varpi \tau_{c}=0.67$. This finding is in agreement with that in the dynamic light scattering problem, i.e., the accuracy of moment equations increases when more moments are included in macroscopic equations. 


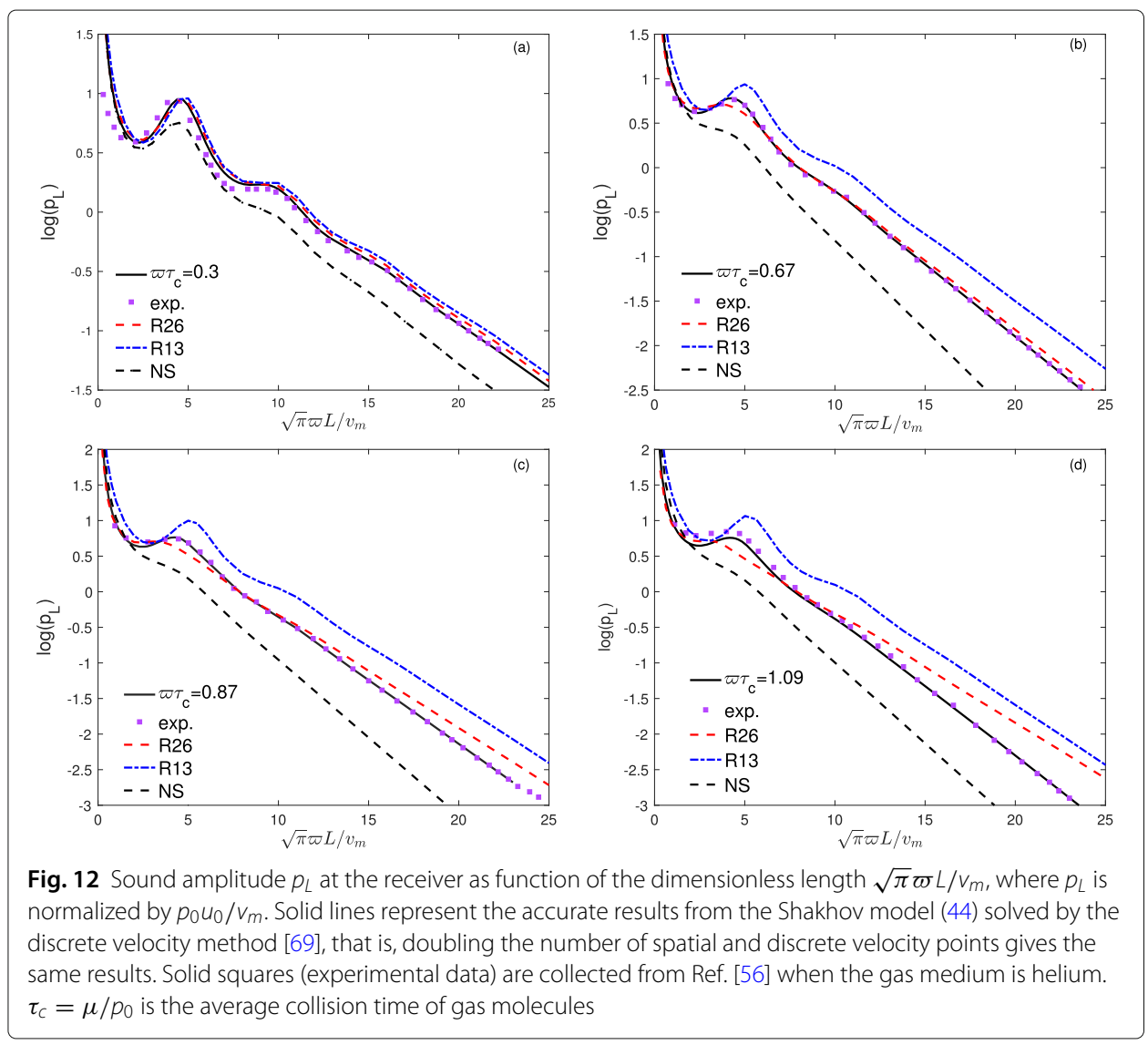

\subsection{Reason of slow convergence of moment systems for highly rarefied flows}

When the boundary condition is involved, it has been shown that the Chapman-Enskog expansion is no longer valid in the boundary layer [65]. Therefore, the order of accuracy in $K n$ may not make too much sense when the gas-surface interaction is important. Nevertheless, it is important to investigate the convergence of moment systems for moderate and highly rarefied gas flows, that is, how many moments should be included to give reasonable prediction of sound pressure? Since the derivation and solving of higher-order moment system is extremely difficult, we solve the Shakhov model (44) using the GaussHermite quadrature of order $N$ instead; this is equivalent to the Grad moment equations where the VDF is expanded by Hermite polynomials up to $N$-th order.

Typical results are depicted in Fig. 13. For moderate value of $\varpi \tau_{c}$ in Fig. 13a, the result of using Gauss-Hermite quadrature of order 8 is more accurate than that of R26 equations. This is because $N=8$ corresponding to Grad moment equations of accuracy $O\left(\mathrm{Kn}^{6}\right)$, one order more accurate than R26 equations. In order to obtain accurate results, however, the VDF has to be expanded into Hermite polynomials to the 20 -th order. We say the convergence is rather slow as increasing $N$ from 10 to 20 only results in marginal improvement of accuracy. This situation becomes more severe when $\varpi \tau_{c}$ increases to 2.72 . From Fig. 13b we see that R13 and R26 moments equations are quite inaccurate; in order to have accurate results, Gauss-Hermite quadrature of order higher than 50 is needed.

The slow convergence of moment systems in describing rarefied gas flows may be explained at the mesoscopic level. To this end we plot the marginal VDF 

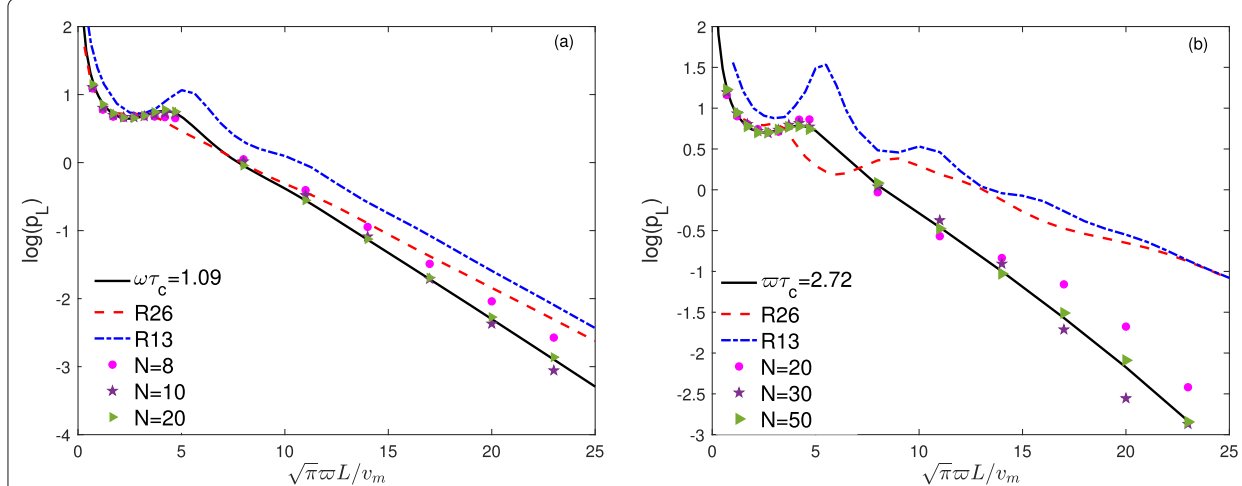

Fig. 13 Convergence test of moment equations: sound amplitude at the receiver as function of the dimensionless length $\sqrt{\pi} \varpi L / V_{m}$. Solid lines represent the accurate results from the Shakhov model (44) solved by the discrete velocity method [69]. Symbols are approximate solutions of the Shakhov model when the molecular velocity space $\mathbf{v}$ is discretized according to Gauss-Hermite quadrature of order $N$; these solutions are equivalent to those of Grad moment equations (having $(N+1)(N+2)(N+3) / 6$ moments) that are accurate up to the order of $\mathrm{Kn}^{\mathrm{N}-2}$

$$
h_{m}=\iint h^{\prime} d v_{2} v_{3}, \quad \text { where } h(\mathbf{v}, t)=\Re\left[h^{\prime}(\mathbf{v}) \exp (i \varpi t)\right],
$$

at the resting receiver when $\sqrt{\pi} \varpi L / v_{m}=20$, and when $\varpi \tau_{c}=1.09$ and 2.72, respectively. Accurate and approximate numerical results of the Shakhov kinetic model by the Gauss-Hermite quadrature are compared in Fig. 14. When $\varpi \tau_{c}=1.09$, the two Knudsen numbers are $K n=0.069$ and $K n_{t}=0.123$, we see from Fig. 14a,b that the VDF is smooth, except it has a huge jump at $v_{1}=0$. This kind of jump (discontinuities) is typical in wallbounded rarefied gas flows. The VDF when $v_{1}<0$ is described by the Gaussian function as per Maxwell's diffuse boundary condition, while that at $v_{1}>0$ deviates from the equilibrium distribution because of the relative large value of $K n_{t}$ so that the system does not have enough time to equilibrate. The Gauss-Hermite quadrature of order $N=8$ cannot predict the gas pressure in Fig. 13a because the VDF in Fig. 14a,b, when $v_{1}>0$, can be well fitted by the Hermite polynomials up to the 8th order. Only the Gauss-Hermite quadrature of order higher than $N=20$ can give reasonable good prediction of macroscopic gas pressure at the receiver. However, from the comparison in VDF we see that this is not enough in capturing accurately the physics at the mesoscopic level. When $\varpi \tau_{c}=2.72$, the two Knudsen numbers are $K n=0.171$ and $K n_{t}=0.306$, the rarefaction effects are even stronger, and the VDF becomes more and more irregular, that is, it not only has jump at $v_{1}=0$, but has rapid variations. To capture this rapid variations, the order of Gauss-Hermite quadrature needs to be very high. From the comparisons in Fig. 13b and Fig. $14 \mathrm{c}, \mathrm{d}$ we see that although Gauss-Hermite quadrature of order $N=50$ can predict the gas pressure at the receiver, it still produces some discrepancy in the mesoscopic VDF.

Thus, we believe that the large discontinuities and rapid variations in the VDF are the underlying reasons for the slow convergence of Grad moment systems, since the smooth Hermite polynomials are not good at resolving these irregular structures in the VDF. In the framework of Gauss-Hermite quadrature, adding more discrete velocity grids is not economic, as these grids will be distributed in the region $\left|v_{1}\right|>3$ where the VDF is zero! In the discrete velocity method, it has already shown that designing numerical quadrature that is more suitable for wall-bounded problems $[2,48,68]$ will greatly increase the 

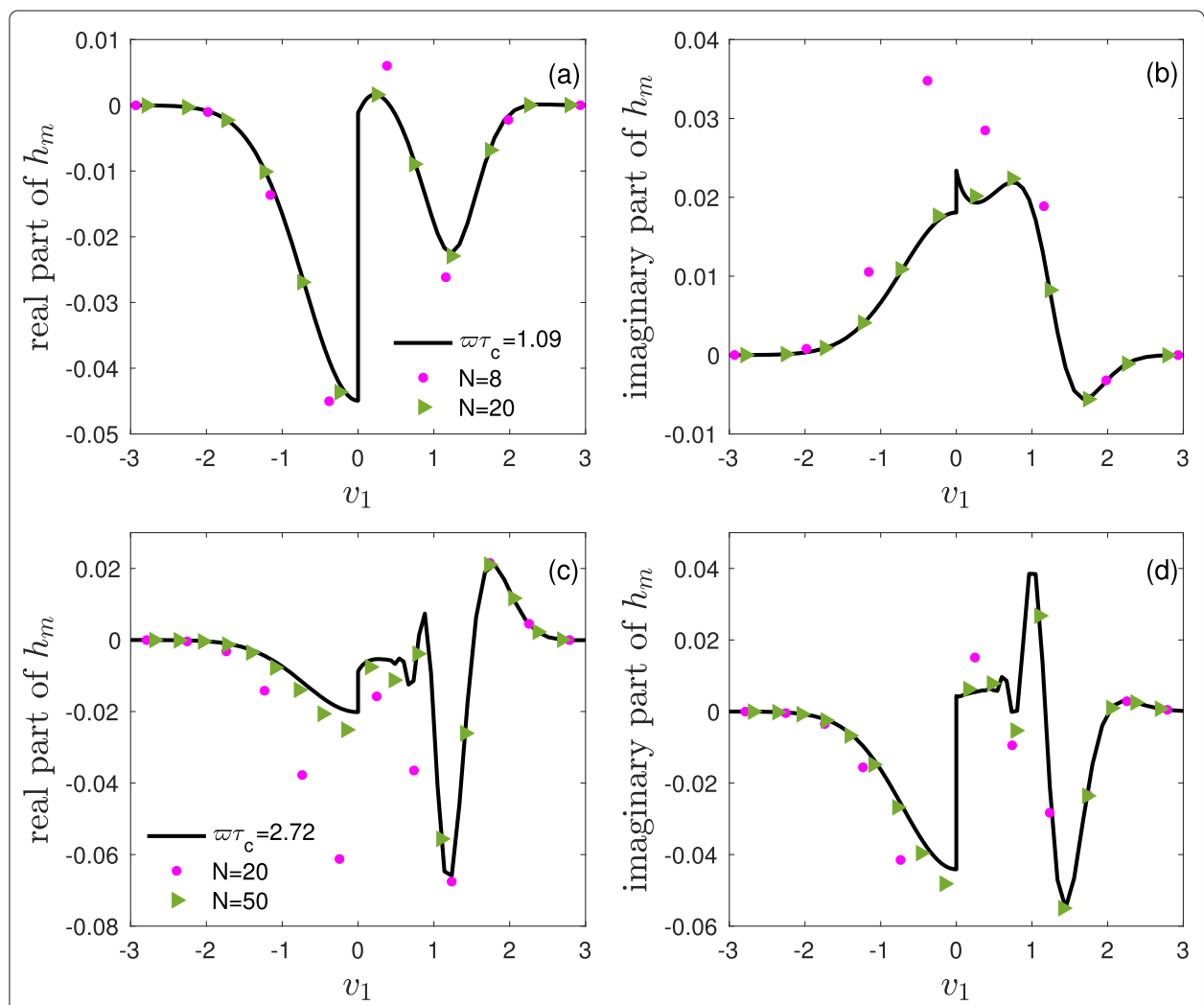

Fig. 14 Marginal VDF (47) at the receiver. $\mathbf{a}, \mathbf{b} \varpi \tau_{c}=1.09$ and $(\mathbf{c}, \mathbf{d}) \varpi \tau_{c}=2.72$. In both cases, $\sqrt{\pi} \varpi L / V_{m}=20$. Solid lines represent the accurate results from the Shakhov model (44) solved by the discrete velocity method [69], while symbols are approximate solutions of the Shakhov model when the molecular velocity space $\mathbf{v}$ is discretized according to the Gauss-Hermite quadrature of order $N$

accuracy while reduce the computational cost. This may hint that to derive more accurate moment equations with limited number of moments, more suitable basis functions rather than the Hermite polynomials should be used.

\section{Conclusions}

In summary, by considering the problems of dynamic light scattering and sound wave propagation, we have assessed the accuracy of more than ten macroscopic equations proposed in the history to describe the rarefied gas dynamics. These equations are derived mainly from one of the three categories: Chapman-Enskog expansion, Grad moment and regularised moment methods, and the argument of "volume diffusion". While the first two categories are based on the Boltzmann equation where the gas dynamics is known as long as the intermolecular potential is determined, the latter has some free parameters which seems cannot be determined from the first-principle physics. In the dynamic light scattering, we found that, (i) higher-order Chapman-Enskog expansion does not necessarily lead to more accurate prediction of rarefied gas dynamics, and since Burnett and super-Burnett equations are not stable, they shall not be used, (ii) macroscopic equations proposed based on the concept of "volume diffusion" do not work well even in the near continuum flow regimes, due to the lack of solid argument from actual micro-physics in these methods, (iii) the moment method can provide reasonable results, and its accuracy always increases when more moments are included. However, for moderate and highly 
rarefied gas flows, a huge number of moments should be included in the moment system as the convergence to true solutions is rather slow. We hope this research could shed some light on how to choose/develop macroscopic equations for rarefied gas dynamics. For example, in the sound propagation problem we have found that the slow convergence of Grad moment systems is due to the ineffectiveness of Hermite polynomials in capturing the large discontinuities and/or large variations in the velocity distribution function. If the basis functions used in the expansion of velocity distribution function are chosen properly, we may be able to construct small-size moment systems to describe the rarefied gas dynamics up to larger values of Knudsen number.

\section{Abbreviations}

G13/26: Grad 13/26; LBE: Linearized Boltzmann Equation; NS: Navier-Stokes; R13/26/35: Regularized 13/26/35; RBS: Rayleigh-Brillouin Scattering; VDF: velocity distribution function

\section{Acknowledgements}

We are grateful to Henning Struchtrup from Victoria University and Zhongzheng Jiang from Zhejiang University for help discussions.

\section{Authors' contributions}

LW designed and wrote the manuscript, while XG solved the sound propagation problem by using NS, R13 and R26 equations. Both authors read and approved the final manuscript.

\section{Funding}

Not applicable.

\section{Availability of data and materials}

All data generated or analyzed during this study are included in this published article.

\section{Competing interests}

Not applicable.

\section{Author details}

${ }^{1}$ Department of Mechanics and Aerospace Engineering, Southern University of Science and Technology, 518055

Shenzhen, China. ${ }^{2}$ Scientific Computing Department, STFC Daresbury Laboratory, WA4 4AD Warrington, UK.

Received: 29 October 2019 Revised: 21 November 2019 Accepted: 13 December 2019

Published online: 08 January 2020

\section{References}

1. Al-Ghoul M, Eu BC (2001) Nonequilibrium partition function in the presence of heat flow. J Chem Phys 115(18):8481. https://doi.org/10.1063/1.1410381

2. Ambruş VE, Sofonea V (2012) High-order thermal lattice Boltzmann models derived by means of Gauss quadrature in the spherical coordinate system. Phys Rev E 86. https://doi.org/10.1103/physreve.86.016708

3. Bardow A, Öttinger HC (2007) Consequences of the Brenner modification to the Navier-Stokes equations for dynamic light scattering. Physica A 373:88-96

4. Bird GA (1994) Molecular Gas Dynamics and the Direct Simulation of Gas Flows. Oxford Science Publications, Oxford University Press Inc, New York

5. Bobylev AV (2006) Instabilities in the Chapman-Enskog expansion and hyperbolic Burnett equations. J Stat Phys 124:371-399

6. Boltzmann L (1872) Weitere Studien über das Wärmegleichgewicht unter Gasmolekülen. Sitz-Ber Akad Wiss Wien 66:275-370

7. Brenner H (2005) Kinematics of volume transport. Physica A 349:11

8. Brenner H (2005) Navier-Stokes revisited. Physica A 349:60

9. Cercignani C (1988) The Boltzmann Equation and its Applications. Springer-Verlag, New York

10. Chapman S (1916) On the law of distribution of molecular velocities, and the theory of viscosity and thermal conduction, in a non-uniform simple monatomic gas. Phil Trans R Soc A 216:538-548

11. Chapman S, Cowling TG (1970) The Mathematical Theory of Non-uniform Gases. Cambridge University Press

12. Christou C, Dadzie SK (2018) On the numerical simulation of rarefied gas flows in micro-channels. J Phys Commun 2:035002

13. Dadzie SK (2013) A thermo-mechanically consistent Burnett regime continuum flow equation without Chapman-Enskog expansion. J Fluid Mech 716:6-11

14. Dadzie SK, Brenner H (2012) Predicting enhanced mass flow rates in gas microchannels using nonkinetic models. Phys Rev E 86:036318

15. Dadzie SK, Reese JM, Mcinnes CR (2008) A continuum model of gas flows with localized density variations. Physica A 387:6079-6094 
16. Durst F (2008) Fluid Mechanics: An Introduction to the Theory of Fluid Flows. Springer, Berlin

17. Enskog D (1917) Kinetische Theorie der Vorgänge in mässig verdünnten Gasen. Uppsala University, Sweden

18. Eu BC (1992) Kinetic thoery and irreversible thermodynamics. Wiley

19. Fan YW (2016) Development and Application of Moment Method in Gas Kinetic Theory. Dissertation, Peking University

20. Garcia-Colin LS, Velasco RM, Uribe FJ (2008) Beyond the Navier-Stokes equations: Burnett hydrodynamics. Phys Rep 465:149-189

21. Gerakis A, Shneider MN, Barker PF (2013) Single-shot coherent Rayleigh-Brillouin scattering using a chirped optical lattice. Opt Letts 38(21):4449

22. Gerakis A, Yeh Y, Shneider MN, Mitrani JM, Stratton BC, Raitses Y (2018) Four-Wave-Mixing approach to in-situ detection of nanoparticles. Phys Rev Appl 9:014031

23. Grad H (1949) On the kinetic theory of rarefied gases. Comm Pure Appl Math 2:331-407

24. Greenshields CJ, Reese JM (2007) The structure of shock waves as a test of Brenner's modifications to the Navier-Stokes equations. J Fluid Mech 580:407-429

25. Greytak TJ, Benedek GB (1966) Spectrum of light scattered from thermal fluctuations in gases. Phys Rev Lett 17:179-182

26. Grinstead JH, Barker PF (2000) Coherent Rayleigh scattering. Phys Rev Lett 85:1222

27. Gu XJ, Emerson DR (2009) A high-order moment approach for capturing non-equilibrium phenomena in the transition regime. J Fluid Mech 636:177-216

28. Gu Z, Ubachs W (2013) Temperature-dependent bulk viscosity of Nitrogen gas determined from spontaneous Rayleigh-Brillouin scattering. Opt Lett 38(7):1110

29. Gu Z, Ubachs W, Marques W, van de Water W (2015) Rayleigh-Brillouin scattering in binary-gas mixtures. Phys Rev Lett 114:243902

30. Gu ZY, Ubachs W, van de Water W (2014) Rayleigh-Brillouin scattering of carbon dioxide. Opt Lett 39:3301

31. Guo ZL, Xu K (2009) Numerical validation of Brenner's hydrodynamic model by force driven Poiseuille flow. Adv Appl Math Mech 3:391-401

32. Hilbert D (1902) Mathematical problems. Bull Am Math Soc 8:437-479

33. Hilbert D (1912) Begrundung der kinetische gastheorie. Math Ann 72:562-577

34. Ivanov MS, Gimelshein SF (1998) Computational hypersonic rarefied flows. Ann Rev Fluid Mech 30:469-505

35. Jiang Z, Zhao W, Chen W, Agarwal RK (2019) Computation of shock wave structure using a simpler set of generalized hydrodynamic equations based on nonlinear coupled constitutive relations. Shock Waves. https://doi. org/10.1007/s00193--018--0876--3

36. Kalempa D, Sharipov $F$ (2012) Sound propagation through a rarefied gas Influence of the gas-surface interaction. Int J Heat Fluid Flow 38:190-199

37. Karlin IV, Gorban AN, Dukek G, Nonnenmacher TF (1998) Dynamic correction to moment approximations. Phys Rev E $57: 1668-1672$

38. Karniadakis G, Beskok A, Aluru N (2005) Microflows and Nanoflows: Fundamentals and Simulation. Springer, New York

39. Ketsdever A, Gimelshein N, Gimelshein S, Selden N (2012) Radiometric phenomena: From the 19th to the 21st century. Vacuum 86:1644-1662

40. Marques Jr W (2008) Is Brenner's modification to the classical Navier-Stokes equations able to describe sound progation in gases?. Chin Phys Lett 4:1355

41. Marques Jr W, Kremer GM (1993) Spectral distribution of scattered light in polyatomic gases. Physica A 197:352-363

42. Maxwell JC (1867) On the dynamical theory of gases. Phil Trans R Soc 157:49-88

43. Maxwell JC (1879) On stresses in rarefied gases arising from inequalities of temperature. Philos Trans R Soc Part 1 170:231-256

44. Mott-Smith HM (1951) The solution of the Boltzmann equation for a shock wave. Phys Rev 82:885-892

45. Muller I, Ruggeri T (1998) Rational Extended Thermodynamics. Springer, New York

46. Myong RS (1999) Thermodynamically consistent hydrodynamic computational models for high-Knudsen-number gas flows. Phys Fluids 11:2788

47. Myong RS (2001) A computational method for Eu's generalized hydrodynamic equations of rarefied and microscale gasdynamics. J Comput Phys 168:47-72

48. Naris S, Valougeorgis D (2005) The driven cavity flow over the whole range of the Knudsen number. Phys Fluids 17:097106

49. Pan X, Shneider MN, Miles RB (2004) Coherent Rayleigh-Brillouin scattering in molecular gases. Phys Rev A 69:033814

50. Pan XG, Shneider MN, Miles RB (2002) Coherent Rayleigh-Brillouin Scattering. Phys Rev Lett 89(18):183001

51. Rana AS, Gupta VK, Struchtrup H (2018) Coupled constitutive relations: a second law based higher-order closure for hydrodynamics. Proc R Soc A 474:20180323

52. Reese JM, Woods LC (1995) A second-order description of shock structure. J Comput Phys 117:240-250

53. Reynolds O (1879) On certain dimensional properties of matter in the gaseous state. Phil Trans R Soc Lond $170: 727-845$

54. Ruggeri T, Sugiyama M (2015) Rational extended thermodynamics beyond the monatomic gas. Springer International Publishing, Switzerland

55. Sambasivam R (2012) Extended Navier-Stokes Equations: Derivations and Applications to Fluid Flow Problems Ph.D. thesis, University of Erlangen-Nuremberg

56. Schotter R (1974) Rarefied gas acoustics in the noble gases. Phys Fluids 17:1163

57. Shakhov EM (1968) Generalization of the Krook kinetic relaxation equation. Fluid Dyn 3:95-96

58. Shan XW, Yuan XF, Chen HD (2006) Kinetic theory representation of hydrodynamics: a way beyond the Navier-Stokes equation. J Fluid Mech 550:413-441

59. Sharipov F, Strapasson J (2012) Ab initio simulation of transport phenomena in rarefied gases. Phys Rev E 86:031130 
60. Shavaliyev MS (1993) Super-Burnett corrections to the stress tensor and the heat flux in a gas of Maxwellian molecules. J Appl Maths Mechs 57:573-576

61. Shneider MN, Gimelshein SF (2013) Application of coherent Rayleigh-Brillouin scattering for in situ nanoparticle and large molecule detection. Appl Phys Lett 102:173109

62. Singh N, Jadhav RS, Agrawal A (2017) Derivation of stable Burnett equations for rarefied gas flows. Phys Rev E 96:013106

63. Sone $Y$ (2002) Kinetic theory and fluid dynamics. Birkhauser, Boston

64. Struchtrup H (2005) Macroscopic Transport Equations for Rarefied Gas Fows: Approximation Methods in Kinetic Theory. Springer, Heidelberg

65. Struchtrup H (2008) Linear kinetic heat transfer: Moment equations, boundary conditions, and Knudsen. Phys A 387:1750-1766

66. Struchtrup H (2011) Resonance in rarefied gases. Cont Mech Theromodyn 34:361-376

67. Struchtrup H, Torrilhon M (2003) Regularization of Grad's 13 moment equations: Derivation and linear analysis. Phys Fluids 15:2668-2690

68. Su W, Lindsay S, Liu HH, Wu L (2017) Comparative study of the discrete velocity and lattice Boltzmann methods for rarefied gas flows through irregular channels. Phys Rev E 96:023309

69. Su W, Zhu LH, Wang P, Zhang YH, Wu L (2019) Can we find steady-state solutions to multiscale rarefied gas flows within dozens of iterations? arXiv:1906:05280

70. Sugawara A, Yip S, Sirovich L (1968) Spectrum of density fluctuations in gases. Phys Fluids 11:925

71. Tenti G, Boley C, Desai R (1974) On the kinetic model description of Rayleigh-Brillouin scattering from molecular gases. Can J Phys 52:285

72. Vieitez MO, van Duijn EJ, Ubachs W, Witschas B, Meijer A, de Wijn AS, Dam NJ, van de Water W (2010) Coherent and spontaneous rayleigh-brillouin scattering in atomic and molecular Coherent and spontaneous Rayleigh-Brillouin scattering in atomic and molecular gases and gas mixtures. Phys Rev A 82:043836

73. Wang-Chang CS (1982) On the theory of the thickness of weak shock waves. Stud Stat Mech 27:29-31

74. Weiss W, Muller I (1995) Light scattering and extended thermodynamics. Cont Mech Theromodyn 7:123-177

75. Witschas B, Lemmerz C, Reitebuch O (2014) Daytime measurements of atmospheric temperature profiles $2-15 \mathrm{~km}$ by lidar utilizing Rayleigh-Brillouin scattering. Opt Lett 39:1972-1975

76. Woods LC (1993) An Introduction to the Kinetic Theory of Gases and Magnetoplasmas

77. Wu L (2016) Sound propagation through a rarefied gas in rectangular channels. Phys Rev E 94:053110

78. Wu L, Reese JM, Zhang YH (2014) Solving the Boltzmann equation by the fast spectral method: application to microflows. J Fluid Mech 746:53-84

79. Wu L, White C, Scanlon TJ, Reese JM, Zhang Y (2014) Coherent Rayleigh-Brillouin scattering: Influence of the intermolecular potential. AIP Conf Proc 1628:648

80. Wu L, White C, Scanlon TJ, Reese JM, Zhang YH (2015) A kinetic model of the Boltzmann equation for nonvibrating polyatomic gases. J Fluid Mech 763:24-50

81. Wu L, Zhang YH (2012) Applicability of the Boltzmann equation for a two-dimensional Fermi gas. Phys Rev A 85:056603

82. Xiao H, Tang K (2017) A unified framework for modeling continuum and rarefied gas flows. Sci Rep 7:13108

83. Zhang J, Fan J (2009) Monte Carlo simulation of thermal fluctuations below the onset of Rayleigh-Benard convection. Phys Rev E 79:053602

84. Zhong X, MacCormack RW, Chapman DR (1993) Stabilization of the Burnett equation and applications to hypersonic flows. AIAA J 31:1036

85. Zhu L, Wu L, Zhang Y, Sharipov F (2019) Ab initio calculation of rarefied flows of helium-neon mixture: classical vs quantum scatterings. Int J Heat Mass Transf 145:118765

\section{Publisher's Note}

Springer Nature remains neutral with regard to jurisdictional claims in published maps and institutional affiliations.

\section{Ready to submit your research? Choose BMC and benefit from:}

- fast, convenient online submission

- thorough peer review by experienced researchers in your field

- rapid publication on acceptance

- support for research data, including large and complex data types

- gold Open Access which fosters wider collaboration and increased citations

- maximum visibility for your research: over $100 \mathrm{M}$ website views per year

At $\mathrm{BMC}$, research is always in progress.

Learn more biomedcentral.com/submissions 